\title{
Asymmetric Conjugate Alkenylation of Enones Catalyzed by
}

\section{Chiral Diols}

\author{
T. Robert Wu and J. Michael Chong* \\ Guelph-Waterloo Centre for Graduate Work in Chemistry and \\ Biochemistry, Department of Chemistry, University of Waterloo, Waterloo, \\ Ontario, Canada N2L 3G1
}

\section{Supporting Information}

\section{Table of Contents}

Proposed model for observed absolute configuration ........................................... S2

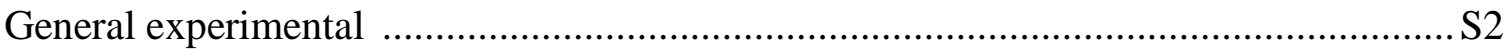

General procedure for the alkenylation of enones …........................................... 3

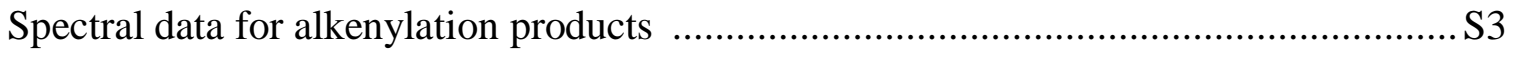

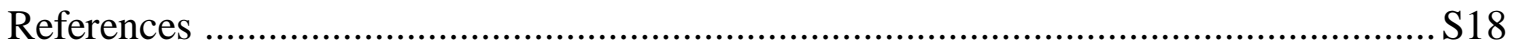

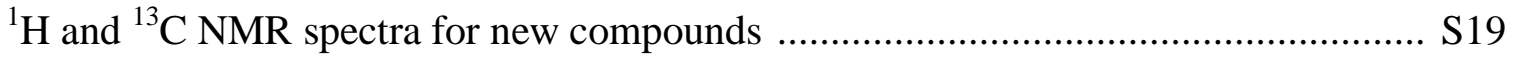




\section{Proposed model for observed absolute configuration}

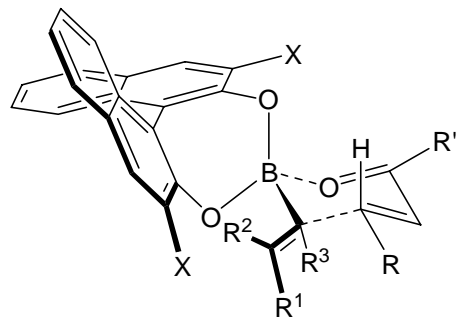

Favored

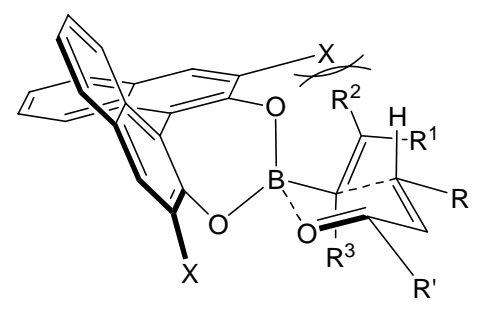

Disfavored<smiles>CC(=O)/C=C/c1ccccc1</smiles>

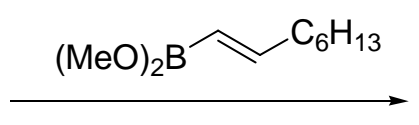

cat. $(R)-\mathbf{4 g}, \mathrm{CH}_{2} \mathrm{Cl}_{2}$

$(R)-\mathbf{4 g}=$<smiles>Oc1c(I)cc2ccccc2c1C(c1ccccc1)c1c(O)c(I)cc2ccccc12</smiles>

$(S, E)$-4-phenyldodec-5-en-2-one $[\alpha]^{25}+1.78\left(\mathrm{c} 1.01, \mathrm{CHCl}_{3}, 98 \%\right.$ ee $)$ lit: $[\alpha]^{27}-1.4$ (c $1.04, \mathrm{CHCl}_{3}, 79 \%$ ee, for $R$ enantiomer) (Oi, S.; Sato, T.; Inoue, Y. Tetrahedron Lett. 2004, 45, 5051-5055)

\section{General Experimental}

All reactions were performed using flame-dried glassware under an argon atmosphere. Dichloromethane was freshly distilled from calcium hydride. Molecular sieves (powder) were activated immediately prior to use. Chiral 3,3'-disubstituted binaphthols were synthesized using procedures from a previous report. ${ }^{1}$ Volatile alkenylboronates (Table 3, entries 1-4 and 6-10) were prepared from the corresponding alkenylboronic acid ${ }^{2-6}$ by treatment with $1: 2.4$ methanol/ $\mathrm{CHCl}_{3}$ in the presence of molecular sieves $3 \AA$ (Dean-Stark apparatus, reflux for 48 hours) and were distilled prior to use. Non-volatile boronates (Table 3, entry 5) were generated from alkenylboronic acids in the presence of 1:2 $\mathrm{MeOH} / \mathrm{CH}_{2} \mathrm{Cl}_{2}$ and molecular sieves $3 \AA$ (room temperature 
for 48 hours) and were used without further purification. ${ }^{1} \mathrm{H}$ and ${ }^{13} \mathrm{C}$ NMR spectra were recorded in $\mathrm{CDCl}_{3}$ at $300 \mathrm{MHz}$ and $75 \mathrm{MHz}$, respectively, unless otherwise specified. Mass spectra were recorded on a Kratos MA890 mass spectrometer using electron impact $(\mathrm{EI}, 70 \mathrm{eV})$ ionization unless otherwise specified. Optical rotations were recorded in cells with $10 \mathrm{~cm}$ path length on a Perkin-Elmer 241 digital polarimeter.

\section{General procedure for the alkenylboration of enones:}

To a mixture of the appropriate enone $(0.30 \mathrm{mmol})$, chiral diol $(0.03 \mathrm{mmol}, 10 \mathrm{~mol} \%)$ and MS $4 \AA$ (300 mg) in $\mathrm{CH}_{2} \mathrm{Cl}_{2}(6 \mathrm{~mL})$, was added alkenylboronate $(0.9 \mathrm{mmol})$ at room temperature. The mixture was brought to reflux for the time indicated in Tables 1-3. Methanol $(0.5 \mathrm{~mL})$ was added after the reaction was cooled to room temperature. Volatiles were removed under high vacuum and the crude material was purified by flash column chromatography on silica gel (hexanes/EtOAc) to give the addition product as a colorless oil.

The enantiomeric purities of the products were determined by HPLC analysis ( $4.6 \mathrm{x}$ $250 \mathrm{~mm}$ ChiralCel OD or ChiralPak AD-H, hexane/i-PrOH $=99.75 / 0.25 \sim 96.75 / 3.25$ $\mathrm{v} / \mathrm{v})$.

(S,E)-1,3-Diphenylundec-4-en-1-one (3a)<smiles>C/C=C/C(CC(=O)c1ccccc1)c1ccccc1</smiles>

$[\alpha]_{589}^{25}+17.1$ (98.6:1.4 er, c 0.34, $\mathrm{CHCl}_{3}$ ); IR (neat): 1687, 1598, 1448, 969, 748, 698

$\mathrm{cm}^{-1} ;{ }^{1} \mathrm{H}$ NMR (300 MHz, $\left.\mathrm{CDCl}_{3}\right): \delta 7.92(\mathrm{~d}, J=7.4 \mathrm{~Hz}, 2 \mathrm{H}), 7.53(\mathrm{t}, J=7.3 \mathrm{~Hz}, 1 \mathrm{H})$, 
$7.43(\mathrm{dd}, J=7.3,7.4 \mathrm{~Hz}, 2 \mathrm{H}), 7.34-7.14(\mathrm{~m}, 5 \mathrm{H}), 5.62(\mathrm{dd}, J=15.3,7.1 \mathrm{~Hz}, 1 \mathrm{H}), 5.43(\mathrm{dt}$, $J=15.3,6.6 \mathrm{~Hz}, 1 \mathrm{H}), 4.07(\mathrm{q}, J=7.1 \mathrm{~Hz}, 1 \mathrm{H}), 3.45-3.27(\mathrm{~m}, 2 \mathrm{H}), 1.96(\mathrm{q}, J=6.6 \mathrm{~Hz}$, $2 \mathrm{H}), 1.35-1.13(\mathrm{~m}, 8 \mathrm{H}), 0.86(\mathrm{t}, J=6.6 \mathrm{~Hz}, 3 \mathrm{H}) ;{ }^{13} \mathrm{C} \mathrm{NMR}\left(75 \mathrm{MHz}, \mathrm{CDCl}_{3}\right): \delta 198.5$, 144.1, 137.3, 132.8, 132.1, 131.1, 128.5, 128.4, 128.0, 127.5, 126.3, 44.8, 43.9, 32.4, 31.6, 29.2, 28.7, 22.5, 14.0; MS m/z (relative intensity): $320\left(\mathrm{M}^{+}, 33\right), 235,\left(\mathrm{M}^{+}-\mathrm{C}_{6} \mathrm{H}_{13}, 6\right), 221$ $\left(\mathrm{M}^{+}-\mathrm{C}_{7} \mathrm{H}_{15}, 23\right)$; HRMS $m / z$ Calcd. for $\mathrm{C}_{23} \mathrm{H}_{28} \mathrm{O}\left(\mathrm{M}^{+}\right)$: 320.2140. Found: 320.2147; the enantiomeric purity was determined by HPLC analysis: (ChiralCel OD, hexane $/ i-\mathrm{PrOH}=$ 99/1, flow rate $=0.5 \mathrm{~mL} / \mathrm{min}), \mathrm{t}_{\mathrm{R}}=18.9 \min (R), \mathrm{t}_{\mathrm{R}}=21.8 \min (S)$.

\section{(S,E)-3-(4-Chlorophenyl)-1-phenylundec-4-en-1-one (3b)}

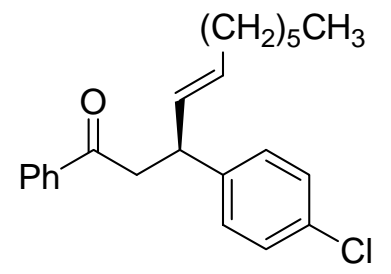

$[\alpha]_{589}^{25}+17.2\left(99.2: 0.8\right.$ er, c $\left.2.63, \mathrm{CHCl}_{3}\right)$; IR (neat): $1688,1597,1491,969,826,752$, $690 \mathrm{~cm}^{-1} ;{ }^{1} \mathrm{H}$ NMR (300 MHz, $\left.\mathrm{CDCl}_{3}\right): \delta 7.90(\mathrm{~d}, J=7.4 \mathrm{~Hz}, 2 \mathrm{H}), 7.53(\mathrm{t}, J=7.3 \mathrm{~Hz}$, 1H), $7.43(\mathrm{dd}, J=7.3,7.4 \mathrm{~Hz}, 2 \mathrm{H}), 7.24(\mathrm{~d}, J=8.5 \mathrm{~Hz}, 2 \mathrm{H}), 7.18(\mathrm{~d}, J=8.5 \mathrm{~Hz}, 2 \mathrm{H})$, $5.58(\mathrm{dd}, J=15.4,7.0 \mathrm{~Hz}, 1 \mathrm{H}), 5.43(\mathrm{dt}, J=15.4,6.6 \mathrm{~Hz}, 1 \mathrm{H}), 4.05(\mathrm{q}, J=7.0 \mathrm{~Hz}, 1 \mathrm{H})$, 3.43-3.23 (m, 2H), $1.96(\mathrm{q}, J=6.6 \mathrm{~Hz}, 2 \mathrm{H}), 1.36-1.15(\mathrm{~m}, 8 \mathrm{H}), 0.86(\mathrm{t}, J=6.6 \mathrm{~Hz}, 3 \mathrm{H})$;

${ }^{13} \mathrm{C}$ NMR (75 MHz, $\left.\mathrm{CDCl}_{3}\right): \delta 198.2,142.6,137.1,133.0,131.9,131.8,131.5,129.0$, 128.6, 128.0, 44.6, 43.2, 32.5, 31.6, 29.2, 28.7, 22.6, 14.0; MS m/z (relative intensity): $354\left(\mathrm{M}^{+}, 22\right), 269\left(\mathrm{M}^{+}-\mathrm{C}_{6} \mathrm{H}_{13}, 3\right), 255\left(\mathrm{M}^{+}-\mathrm{C}_{7} \mathrm{H}_{15}, 11\right)$; HRMS $m / z$ Calcd. for $\mathrm{C}_{23} \mathrm{H}_{27} \mathrm{ClO}$ $\left(\mathrm{M}^{+}\right)$: 354.1750. Found: 354.1758; the enantiomeric purity was determined by HPLC 
analysis: $($ ChiralPak AD-H, hexane $/ i-\mathrm{PrOH}=98.75 / 1.25$, flow rate $=1.0 \mathrm{~mL} / \mathrm{min}), \mathrm{t}_{\mathrm{R}}=$ $8.6 \min (R), \mathrm{t}_{\mathrm{R}}=9.6 \min (S)$.

(S,E)-3-(4-Methoxyphenyl)-1-phenylundec-4-en-1-one (3c)<smiles>C/C=C/C(CC(=O)c1ccccc1)c1ccc(OC)cc1</smiles>

$[\alpha]_{589}^{25}+11.0\left(99: 1\right.$ er, c 1.9, $\left.\mathrm{CHCl}_{3}\right) ; \mathrm{IR}$ (neat): 1687, 1610, 1511, 1448, 1246, 969, 829, 756, $690 \mathrm{~cm}^{-1} ;{ }^{1} \mathrm{H}$ NMR $\left(300 \mathrm{MHz}, \mathrm{CDCl}_{3}\right): \delta 7.91(\mathrm{~d}, J=7.4 \mathrm{~Hz}, 2 \mathrm{H}), 7.53(\mathrm{t}, J=7.3$ $\mathrm{Hz}, 1 \mathrm{H}), 7.42(\mathrm{dd}, J=7.3,7.4 \mathrm{~Hz}, 2 \mathrm{H}), 7.16(\mathrm{~d}, J=8.5 \mathrm{~Hz}, 2 \mathrm{H}), 6.82(\mathrm{~d}, J=8.5 \mathrm{~Hz}, 2 \mathrm{H})$, $5.60(\mathrm{dd}, J=15.4,7.0 \mathrm{~Hz}, 1 \mathrm{H}), 5.43(\mathrm{dt}, J=15.4,6.6 \mathrm{~Hz}, 1 \mathrm{H}), 4.01(\mathrm{q}, J=7.0 \mathrm{~Hz}, 1 \mathrm{H})$, $3.76(\mathrm{~s}, 3 \mathrm{H}), 3.41-3.22(\mathrm{~m}, 2 \mathrm{H}), 1.95(\mathrm{q}, J=6.6 \mathrm{~Hz}, 2 \mathrm{H}), 1.34-1.13(\mathrm{~m}, 8 \mathrm{H}), 0.86(\mathrm{t}, J=$ $6.6 \mathrm{~Hz}, 3 \mathrm{H}) ;{ }^{13} \mathrm{C}$ NMR $\left(75 \mathrm{MHz}, \mathrm{CDCl}_{3}\right): \delta 198.7,158.0,137.3,136.1,132.8,132.4$, 130.7, 128.4, 128.0, 113.8, 55.2, 44.9, 43.1, 32.4, 31.6, 29.2, 28.7, 22.5, 14.0; MS m/z (relative intensity): $350\left(\mathrm{M}^{+}, 84\right), 265\left(\mathrm{M}^{+}-\mathrm{C}_{6} \mathrm{H}_{13}, 16\right), 251\left(\mathrm{M}^{+}-\mathrm{C}_{7} \mathrm{H}_{15}, 21\right), 231\left(\mathrm{M}^{+}-\right.$ $\left.\mathrm{C}_{6} \mathrm{H}_{5} \mathrm{COCH}_{2}, 86\right)$; HRMS $\mathrm{m} / z$ Calcd. for $\mathrm{C}_{24} \mathrm{H}_{30} \mathrm{O}_{2}\left(\mathrm{M}^{+}\right)$: 350.2246. Found: 350.2242 ; the enantiomeric purity was determined by HPLC analysis: (ChiralPak AD-H, hexane/i$\operatorname{PrOH}=99.25 / 0.75$, flow rate $=1.0 \mathrm{~mL} / \mathrm{min}), \mathrm{t}_{\mathrm{R}}=22.3 \min (R), \mathrm{t}_{\mathrm{R}}=24.8 \min (S)$.

$(S, E)-1-P h e n y l-3-p$-tolylundec-4-en-1-one (3d)<smiles>C/C=C/C(CC(=O)c1ccccc1)c1ccc(C)cc1</smiles> 
$[\alpha]_{589}^{25}+15.0\left(99.4: 0.6\right.$ er, c 1.50, $\left.\mathrm{CHCl}_{3}\right)$; IR (neat): 1687, 1597, 1448, 968, 815, 755, $690 \mathrm{~cm}^{-1} ;{ }^{1} \mathrm{H}$ NMR $\left(300 \mathrm{MHz}, \mathrm{CDCl}_{3}\right): \delta 7.93(\mathrm{~d}, J=7.4 \mathrm{~Hz}, 2 \mathrm{H}), 7.54(\mathrm{t}, J=7.3 \mathrm{~Hz}$, $1 \mathrm{H}), 7.44(\mathrm{dd}, J=7.3,7.4 \mathrm{~Hz}, 2 \mathrm{H}), 7.17(\mathrm{~d}, J=8.1 \mathrm{~Hz}, 2 \mathrm{H}), 7.11(\mathrm{~d}, J=8.1 \mathrm{~Hz}, 2 \mathrm{H})$, $5.62(\mathrm{dd}, J=15.3,7.1 \mathrm{~Hz}, 1 \mathrm{H}), 5.43(\mathrm{dt}, J=15.3,6.6 \mathrm{~Hz}, 1 \mathrm{H}), 4.05(\mathrm{q}, J=7.1 \mathrm{~Hz}, 1 \mathrm{H})$, $3.44-3.26(\mathrm{~m}, 2 \mathrm{H}), 2.31(\mathrm{~s}, 3 \mathrm{H}), 1.97(\mathrm{q}, J=6.6 \mathrm{~Hz}, 2 \mathrm{H}), 1.36-1.17(\mathrm{~m}, 8 \mathrm{H}), 0.87(\mathrm{t}, J=$ $6.6 \mathrm{~Hz}, 3 \mathrm{H}) ;{ }^{13} \mathrm{C} \mathrm{NMR}\left(75 \mathrm{MHz}, \mathrm{CDCl}_{3}\right): \delta 198.7,141.1,137.4,135.8,132.8,132.4$, $130.9,129.2,128.5,128.1,127.4,44.9,43.5,32.5,31.7,29.3,28.8,22.6,21.0,14.0, ; \mathrm{MS}$ $m / z$ (relative intensity): $334\left(\mathrm{M}^{+}, 45\right), 249\left(\mathrm{M}^{+}-\mathrm{C}_{6} \mathrm{H}_{13}, 9\right), 235\left(\mathrm{M}^{+}-\mathrm{C}_{7} \mathrm{H}_{15}, 32\right)$; HRMS $m / z$ Calcd. for $\mathrm{C}_{24} \mathrm{H}_{30} \mathrm{O}\left(\mathrm{M}^{+}\right)$: 334.2297. Found: 334.2299; the enantiomeric purity was determined by HPLC analysis: (ChiralPak AD-H, hexane/ $i-\mathrm{PrOH}=99.75 / 0.25$, flow rate $=1.0 \mathrm{~mL} / \mathrm{min}), \mathrm{t}_{\mathrm{R}}=21.3 \min (S), \mathrm{t}_{\mathrm{R}}=25.3 \min (R)$.

\section{(S,E)-1-Phenyl-3-o-tolylundec-4-en-1-one (3e)}

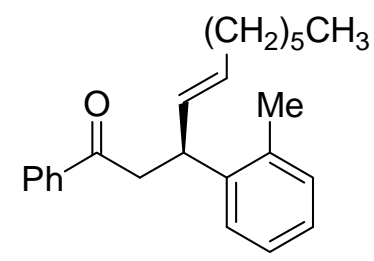

$[\alpha]_{589}^{25}+35.1\left(>99.5: 0.5 \mathrm{er}, \mathrm{c} 4.3, \mathrm{CHCl}_{3}\right) ; \mathrm{IR}$ (neat): 1688, 1598, 1448, 968, 754, 690 $\mathrm{cm}^{-1} ;{ }^{1} \mathrm{H}$ NMR $\left(300 \mathrm{MHz}, \mathrm{CDCl}_{3}\right): \delta 7.95(\mathrm{~d}, J=7.4 \mathrm{~Hz}, 2 \mathrm{H}), 7.55(\mathrm{t}, J=7.3 \mathrm{~Hz}, 1 \mathrm{H})$, $7.44(\mathrm{dd}, J=7.3,7.4 \mathrm{~Hz}, 2 \mathrm{H}), 7.25-7.06(\mathrm{~m}, 4 \mathrm{H}), 5.56(\mathrm{dd}, J=15.3,6.9 \mathrm{~Hz}, 1 \mathrm{H}), 5.36$ (dt, $J=15.3,6.6 \mathrm{~Hz}, 1 \mathrm{H}), 4.32(\mathrm{q}, J=6.9 \mathrm{~Hz}, 1 \mathrm{H}), 3.49-3.26(\mathrm{~m}, 2 \mathrm{H}), 2.39(\mathrm{~s}, 3 \mathrm{H}), 1.94$ $(\mathrm{q}, J=6.6 \mathrm{~Hz}, 2 \mathrm{H}), 1.35-1.15(\mathrm{~m}, 8 \mathrm{H}), 0.86(\mathrm{t}, J=6.6 \mathrm{~Hz}, 3 \mathrm{H}) ;{ }^{13} \mathrm{C} \mathrm{NMR}(75 \mathrm{MHz}$, $\left.\mathrm{CDCl}_{3}\right): \delta 198.6,142.1,137.3,135.9,132.9,131.8,131.0,130.5,128.5,128.1,126.3$, 126.1, 44.2, 39.2, 32.5, 31.7, 29.3, 28.8, 22.6, 19.6, 14.1; MS m/z (relative intensity): 334 
$\left(\mathrm{M}^{+}, 45\right), 249\left(\mathrm{M}^{+}-\mathrm{C}_{6} \mathrm{H}_{13}, 9\right), 235\left(\mathrm{M}^{+}-\mathrm{C}_{7} \mathrm{H}_{15}, 32\right)$; HRMS $m / z$ Calcd. for $\mathrm{C}_{24} \mathrm{H}_{30} \mathrm{O}\left(\mathrm{M}^{+}\right)$: 334.2297. Found: 334.2299; the enantiomeric purity was determined by HPLC analysis: $($ ChiralPak AD-H, hexane $/ i-\mathrm{PrOH}=99.75 / 0.25$, flow rate $=1.0 \mathrm{~mL} / \mathrm{min}), \mathrm{t}_{\mathrm{R}}=12.3 \mathrm{~min}$ $(S), \mathrm{t}_{\mathrm{R}}=14.2 \min (R)$.

\section{(S,E)-3-(Naphthalen-1-yl)-1-phenylundec-4-en-1-one (3f)}

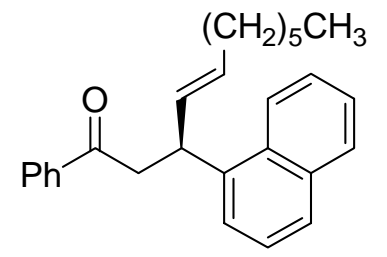

$[\alpha]^{25} 589-4.04$ (>99.5:0.5 er, c 2.35, $\mathrm{CHCl}_{3}$ ); IR (neat): 1686, 1597, 1448, 969, 794, 777, 755, 690, $668 \mathrm{~cm}^{-1} ;{ }^{1} \mathrm{H}$ NMR $\left(300 \mathrm{MHz}, \mathrm{CDCl}_{3}\right): \delta 8.23(\mathrm{~d}, J=6.6 \mathrm{~Hz}, 1 \mathrm{H}), 7.98(\mathrm{~d}, J=$ $8.0 \mathrm{~Hz}, 2 \mathrm{H}), 7.87(\mathrm{~d}, J=8.0 \mathrm{~Hz}, 1 \mathrm{H}), 7.75(\mathrm{~d}, J=4.7 \mathrm{~Hz}, 1 \mathrm{H}), 7.59-7.40(\mathrm{~m}, 7 \mathrm{H}), 5.78$ $(\mathrm{dd}, J=15.4,6.6 \mathrm{~Hz}, 1 \mathrm{H}), 5.50(\mathrm{dt}, J=15.4,6.6 \mathrm{~Hz}, 1 \mathrm{H}), 5.00(\mathrm{~m}, 1 \mathrm{H}), 3.64(\mathrm{dd}, J=$ $16.8,9.0 \mathrm{~Hz}, 1 \mathrm{H}), 3.42(\mathrm{dd}, J=16.8,4.7 \mathrm{~Hz}, 1 \mathrm{H}), 2.00(\mathrm{q}, J=6.6 \mathrm{~Hz}, 2 \mathrm{H}), 1.37-1.16(\mathrm{~m}$, $8 \mathrm{H}), 0.87(\mathrm{t}, J=6.6 \mathrm{~Hz}, 3 \mathrm{H}) ;{ }^{13} \mathrm{C} \mathrm{NMR}\left(75 \mathrm{MHz}, \mathrm{CDCl}_{3}\right): \delta 198.5,140.4,137.3,134.1$, 133.0, 131.7, 131.4, 128.9, 128.6, 128.1, 127.1, 126.0, 125.5, 125.4, 124.1, 123.7, 44.4, 38.6, 32.6, 31.7, 29.3, 28.8, 22.6, 14.1; MS $\mathrm{m} / z$ (relative intensity): $370\left(\mathrm{M}^{+}, 79\right), 285$ $\left(\mathrm{M}^{+}-\mathrm{C}_{6} \mathrm{H}_{13}, 4\right), 271\left(\mathrm{M}^{+}-\mathrm{C}_{7} \mathrm{H}_{15}, 9\right), 251\left(\mathrm{M}^{+}-\mathrm{C}_{6} \mathrm{H}_{5} \mathrm{COCH}_{2}, 31\right)$; HRMS $\mathrm{m} / z$ Calcd. for $\mathrm{C}_{27} \mathrm{H}_{30} \mathrm{O}\left(\mathrm{M}^{+}\right)$: 370.2297 . Found: 370.2298 ; the enantiomeric purity was determined by HPLC analysis: $($ ChiralCel OD, hexane $/ i-\mathrm{PrOH}=99 / 1$, flow rate $=0.5 \mathrm{~mL} / \mathrm{min}), \mathrm{t}_{\mathrm{R}}=$ $19.6 \min (R), \mathrm{t}_{\mathrm{R}}=29.2 \min (S)$. 


\section{(S,E)-3-(Furan-2-yl)-1-phenylundec-4-en-1-one (3g)}

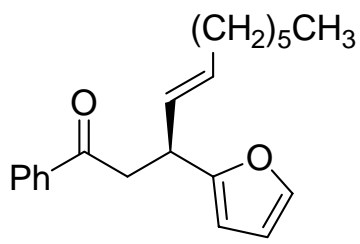

$[\alpha]_{589}^{25}+42.6\left(98.5: 1.5\right.$ er, c $\left.1.32, \mathrm{CHCl}_{3}\right)$; IR (neat): 1689, 1597, 1449, 1010, 968, 755, $730,690 \mathrm{~cm}^{-1} ;{ }^{1} \mathrm{H}$ NMR $\left(300 \mathrm{MHz}, \mathrm{CDCl}_{3}\right): \delta 7.93(\mathrm{~d}, J=7.4 \mathrm{~Hz}, 2 \mathrm{H}), 7.54(\mathrm{t}, J=7.3$ $\mathrm{Hz}, 1 \mathrm{H}), 7.43(\mathrm{dd}, J=7.3,7.4 \mathrm{~Hz}, 2 \mathrm{H}), 7.29(\mathrm{~d}, J=1.8 \mathrm{~Hz}, 1 \mathrm{H}), 6.26(\mathrm{dd}, J=1.8,2.9 \mathrm{~Hz}$, $1 \mathrm{H}), 6.03(\mathrm{~d}, J=2.9 \mathrm{~Hz}, 1 \mathrm{H}), 5.60-5.43(\mathrm{~m}, 2 \mathrm{H}), 4.14(\mathrm{~m}, 1 \mathrm{H}), 3.43(\mathrm{dd}, J=16.4,6.3 \mathrm{~Hz}$, 1H), $3.26(\mathrm{dd}, J=16.4,7.8 \mathrm{~Hz}, 1 \mathrm{H}), 1.97(\mathrm{q}, J=6.6 \mathrm{~Hz}, 2 \mathrm{H}), 1.36-1.15(\mathrm{~m}, 8 \mathrm{H}), 0.85(\mathrm{t}$, $J=6.6 \mathrm{~Hz}, 3 \mathrm{H}) ;{ }^{13} \mathrm{C} \mathrm{NMR}\left(75 \mathrm{MHz}, \mathrm{CDCl}_{3}\right): \delta 198.1,156.9,141.2,137.2,133.0,132.7$, $129.1,128.6,128.1,110.1,105.0,42.6,37.9,32.4,31.7,29.1,28.7,22.6,14.1 ; \mathrm{MS} m / z$ (relative intensity): $310\left(\mathrm{M}^{+}, 23\right), 225\left(\mathrm{M}^{+}-\mathrm{C}_{6} \mathrm{H}_{13}, 6\right), 211\left(\mathrm{M}^{+}-\mathrm{C}_{7} \mathrm{H}_{15}, 7\right)$; HRMS m/z Calcd. for $\mathrm{C}_{21} \mathrm{H}_{26} \mathrm{O}_{2}\left(\mathrm{M}^{+}\right)$: 310.1933. Found: 310.1941; the enantiomeric purity was determined by HPLC analysis: (ChiralPak AD-H, hexane/ $i-\mathrm{PrOH}=99.75 / 0.25$, flow rate $=1.0 \mathrm{~mL} / \mathrm{min}), \mathrm{t}_{\mathrm{R}}=18.2 \min (R), \mathrm{t}_{\mathrm{R}}=22.1 \min (S)$.

$(S, E)-1-P h e n y l-3-((E)-s t y r y l) u n d e c-4-e n-1-o n e ~(3 h)$

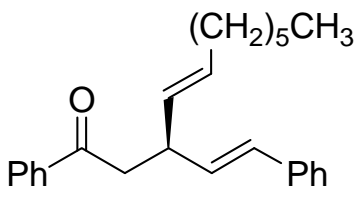

$[\alpha]_{589}^{25}+9.4\left(99: 1\right.$ er, c 6.0, $\left.\mathrm{CHCl}_{3}\right)$; IR (neat): 1687, 1598, 1448, 1010, 967, 751, 691, $668 \mathrm{~cm}^{-1} ;{ }^{1} \mathrm{H}$ NMR $\left(300 \mathrm{MHz}, \mathrm{CDCl}_{3}\right): \delta 7.95(\mathrm{~d}, J=7.4 \mathrm{~Hz}, 2 \mathrm{H}), 7.55(\mathrm{t}, J=7.2 \mathrm{~Hz}$, 1H), 7.45 (dd, $J=7.2,7.4 \mathrm{~Hz}, 2 \mathrm{H}), 7.37-7.14(\mathrm{~m}, 5 \mathrm{H}), 6.42(\mathrm{~d}, J=16.0 \mathrm{~Hz}, 1 \mathrm{H}), 6.22$ $(\mathrm{dd}, J=16.0,7.1 \mathrm{~Hz}, 1 \mathrm{H}), 5.60-5.42(\mathrm{~m}, 2 \mathrm{H}), 3.64(\mathrm{~m}, 1 \mathrm{H}), 3.25-3.08(\mathrm{~m}, 2 \mathrm{H}), 2.00(\mathrm{q}, J$ 
$=6.6 \mathrm{~Hz}, 2 \mathrm{H}), 1.39-1.17(\mathrm{~m}, 8 \mathrm{H}), 0.87(\mathrm{t}, J=6.6 \mathrm{~Hz}, 3 \mathrm{H}) ;{ }^{13} \mathrm{C} \mathrm{NMR}\left(75 \mathrm{MHz}, \mathrm{CDCl}_{3}\right): \delta$ 198.7, 137.5, 137.4, 133.0, 132.4, 131.7, 131.0, 129.8, 128.6, 128.5, 128.2, 127.1, 126.2, 43.9, 41.5, 32.6, 31.7, 29.3, 28.8, 22.6, 14.1; MS m/z (relative intensity): $346\left(\mathrm{M}^{+}, 29\right)$, $261\left(\mathrm{M}^{+}-\mathrm{C}_{6} \mathrm{H}_{13}, 8\right), 247\left(\mathrm{M}^{+}-\mathrm{C}_{7} \mathrm{H}_{15}, 9\right), 227\left(\mathrm{M}^{+}-\mathrm{PhCOCH}_{2}, 9\right), 226\left(\mathrm{M}^{+}-\mathrm{PhCOCH}_{3}, 36\right)$; HRMS $\mathrm{m} / \mathrm{z}$ Calcd. for $\mathrm{C}_{25} \mathrm{H}_{30} \mathrm{O}\left(\mathrm{M}^{+}\right)$: 346.2297. Found: 346.2296; the enantiomeric purity was determined by HPLC analysis: (ChiralPak AD-H, hexane/ $i-\mathrm{PrOH}=99.75 / 0.25$, flow rate $=1.0 \mathrm{~mL} / \mathrm{min}), \mathrm{t}_{\mathrm{R}}=23.4 \min (S), \mathrm{t}_{\mathrm{R}}=25.4 \min (R)$.

$(S, E)$-1-Phenyl-3-((E)-1-phenylprop-1-en-2-yl)undec-4-en-1-one (3i)

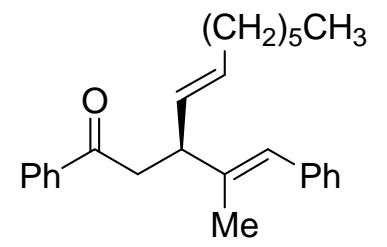

$[\alpha]_{589}^{25}-6.2$ (99.2:0.8 er, c 5.4, $\left.\mathrm{CHCl}_{3}\right)$; IR (neat): 1686, 1598, 1261, 1020, 800, 753, 698 $\mathrm{cm}^{-1} ;{ }^{1} \mathrm{H}$ NMR (300 MHz, $\left.\mathrm{CDCl}_{3}\right): \delta 7.94(\mathrm{~d}, J=7.3 \mathrm{~Hz}, 2 \mathrm{H}), 7.54(\mathrm{t}, J=7.3 \mathrm{~Hz}, 1 \mathrm{H})$, $7.45(\mathrm{t}, J=7.3 \mathrm{~Hz}, 2 \mathrm{H}), 7.32-7.11(\mathrm{~m}, 5 \mathrm{H}), 6.29(\mathrm{~s}, 1 \mathrm{H}), 5.54-5.37(\mathrm{~m}, 2 \mathrm{H}), 3.50(\mathrm{q}, J=$ $6.6 \mathrm{~Hz}, 1 \mathrm{H}), 3.29-3.09(\mathrm{~m}, 2 \mathrm{H}), 2.02-1.90(\mathrm{~m}, 2 \mathrm{H}), 1.84(\mathrm{~s}, 3 \mathrm{H}), 1.37-1.13(\mathrm{~m}, 8 \mathrm{H}), 0.85$ $(\mathrm{t}, J=6.6 \mathrm{~Hz}, 3 \mathrm{H}) ;{ }^{13} \mathrm{C}$ NMR $\left(75 \mathrm{MHz}, \mathrm{CDCl}_{3}\right): \delta 199.2,140.0,138.2,137.5,132.9$, $131.8,130.8,128.9,128.6,128.1,128.0,126.0,125.4,47.9,42.2,32.5,31.7,29.3,28.8$, 22.6, 16.1, 14.1; MS m/z (relative intensity): $360\left(\mathrm{M}^{+}, 29\right), 241\left(\mathrm{M}^{+}-\mathrm{PhCOCH}_{2}, 28\right), 240$ $\left(\mathrm{M}^{+}-\mathrm{PhCOCH}_{3}, 89\right)$; HRMS $\mathrm{m} / z$ Calcd. for $\mathrm{C}_{26} \mathrm{H}_{32} \mathrm{O}\left(\mathrm{M}^{+}\right)$: 360.2453. Found: 360.2465; the enantiomeric purity was determined by HPLC analysis: (ChiralPak AD-H, hexane/i$\operatorname{PrOH}=99.75 / 0.25$, flow rate $=1.0 \mathrm{~mL} / \mathrm{min}), \mathrm{t}_{\mathrm{R}}=14.3 \min (S), \mathrm{t}_{\mathrm{R}}=17.3 \mathrm{~min}(R)$. 


\section{$(S, E)-3-M e t h y l-1-p h e n y l u n d e c-4-e n-1-o n e ~(3 j)$}

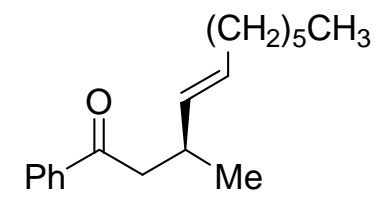

$[\alpha]_{589}^{25}+13.8\left(98: 2 \mathrm{er}, \mathrm{c} 1.96, \mathrm{CHCl}_{3}\right)$; IR (neat): 1687, 1598, 1449, 968, 800, 751, 693 $\mathrm{cm}^{-1} ;{ }^{1} \mathrm{H}$ NMR $\left(500 \mathrm{MHz}, \mathrm{CDCl}_{3}\right): \delta 7.96(\mathrm{~d}, J=7.4 \mathrm{~Hz}, 2 \mathrm{H}), 7.56(\mathrm{t}, J=7.4 \mathrm{~Hz}, 1 \mathrm{H})$, $7.46(\mathrm{t}, J=7.4 \mathrm{~Hz}, 2 \mathrm{H}), 5.45-5.37(\mathrm{~m}, 2 \mathrm{H}), 3.05-2.80(\mathrm{~m}, 3 \mathrm{H}), 1.99-1.93(\mathrm{~m}, 2 \mathrm{H}), 1.40-$ $1.20(\mathrm{~m}, 8 \mathrm{H}), 1.09(\mathrm{~d}, J=6.3 \mathrm{~Hz}, 3 \mathrm{H}), 0.89(\mathrm{t}, J=6.9 \mathrm{~Hz}, 3 \mathrm{H}) ;{ }^{13} \mathrm{C} \mathrm{NMR}(125 \mathrm{MHz}$, $\left.\mathrm{CDCl}_{3}\right): \delta 199.7,137.5,134.5,132.8,129.3,128.5,128.1,124.6,45.9,33.1,32.5,31.7$ 29.4, 28.8, 22.6, 20.6, 14.1; MS $m / z$ (relative intensity): $258\left(\mathrm{M}^{+}, 21\right), 243\left(\mathrm{M}^{+}-\mathrm{CH}_{3}, 5\right)$; HRMS $m / z$ Calcd. for $\mathrm{C}_{18} \mathrm{H}_{26} \mathrm{O}\left(\mathrm{M}^{+}\right)$: 258.1984. Found: 258.1982; the enantiomeric purity was determined by HPLC analysis: (ChiralPak AD-H, hexane/i-PrOH = 99.75/0.25, flow rate $=0.5 \mathrm{~mL} / \mathrm{min}), \mathrm{t}_{\mathrm{R}}=15.0 \mathrm{~min}(S), \mathrm{t}_{\mathrm{R}}=16.4 \min (R)$.

\section{$(S, E)-3-H e x y l-1-p h e n y l u n d e c-4-e n-1-o n e ~(3 k)$}

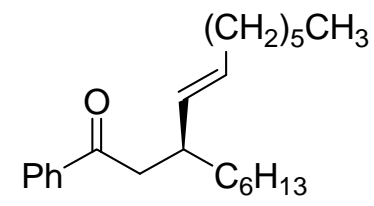

$[\alpha]^{25}{ }_{589}+3.68$ (99.2:0.8 er, c 0.95, $\left.\mathrm{CHCl}_{3}\right)$; IR (neat): 1688, 1598, 1448, 968, 800, 751, $690 \mathrm{~cm}^{-1} ;{ }^{1} \mathrm{H}$ NMR $\left(300 \mathrm{MHz}, \mathrm{CDCl}_{3}\right): \delta 7.90(\mathrm{~d}, J=7.5 \mathrm{~Hz}, 2 \mathrm{H}), 7.52(\mathrm{t}, J=7.2 \mathrm{~Hz}$, 1H), $7.42(\mathrm{dd}, J=7.2,7.5 \mathrm{~Hz}, 2 \mathrm{H}), 5.34(\mathrm{dt}, J=15.3,6.5 \mathrm{~Hz}, 1 \mathrm{H}), 5.19$ (dd, $J=15.3,8.4$ $\mathrm{Hz}, 1 \mathrm{H}), 2.98-2.82(\mathrm{~m}, 2 \mathrm{H}), 2.63(\mathrm{~m}, 1 \mathrm{H}), 1.90(\mathrm{q}, J=6.5 \mathrm{~Hz}, 2 \mathrm{H}), 1.68(\mathrm{~m}, 1 \mathrm{H}), 1.49-$ $1.11(\mathrm{~m}, 18 \mathrm{H}), 0.90-0.79(\mathrm{~m}, 6 \mathrm{H}) ;{ }^{13} \mathrm{C} \mathrm{NMR}\left(75 \mathrm{MHz}, \mathrm{CDCl}_{3}\right): \delta 200,137.5,132.9$, 
$132.7,131.1,128.5,128.1,77.4,77.0,76.6,44.7,39.1,35.3,32.5,31.8,31.7,29.4,29.3$, 28.7, 27.1, 22.6, 22.6, 14.1; MS $m / z$ (relative intensity): $328\left(\mathrm{M}^{+}, 11\right), 257\left(\mathrm{M}^{+}-\mathrm{C}_{5} \mathrm{H}_{11}, 6\right)$, $243\left(\mathrm{M}^{+}-\mathrm{C}_{6} \mathrm{H}_{13}, 35\right)$; HRMS $m / z$ Calcd. for $\mathrm{C}_{23} \mathrm{H}_{36} \mathrm{O}\left(\mathrm{M}^{+}\right)$: 328.2766. Found: 328.2765; the enantiomeric purity was determined by HPLC analysis: (ChiralPak AD-H, hexane/i$\operatorname{PrOH}=99.75 / 0.25$, flow rate $=1.0 \mathrm{~mL} / \mathrm{min}), \mathrm{t}_{\mathrm{R}}=7.4 \min (S), \mathrm{t}_{\mathrm{R}}=8.9 \mathrm{~min}(R)$.

$(R, E)-3-I s o p r o p y l-1-p h e n y l u n d e c-4-e n-1-o n e ~(31)$

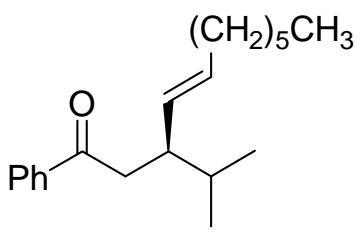

$[\alpha]_{589}^{25}+15.9$ (97:3 er, c 1.04, $\left.\mathrm{CHCl}_{3}\right)$; IR (neat): 1688, 1598, 1449, 970, 750, $691 \mathrm{~cm}^{-1}$; ${ }^{1} \mathrm{H}$ NMR $\left(300 \mathrm{MHz}, \mathrm{CDCl}_{3}\right): \delta 7.89(\mathrm{~d}, J=7.4 \mathrm{~Hz}, 2 \mathrm{H}), 7.51(\mathrm{t}, J=7.3 \mathrm{~Hz}, 1 \mathrm{H}), 7.42(\mathrm{dd}$, $J=7.3,7.4 \mathrm{~Hz}, 2 \mathrm{H}), 5.15-5.35(\mathrm{~m}, 2 \mathrm{H}), 3.01-2.85(\mathrm{~m}, 2 \mathrm{H}), 2.52(\mathrm{~m}, 1 \mathrm{H}), 1.89(\mathrm{q}, J=6.5$ Hz, 2H), $1.68(\mathrm{~m}, 1 \mathrm{H}), 1.30-1.11(\mathrm{~m}, 8 \mathrm{H}), 0.90(\mathrm{~d}, J=6.9 \mathrm{~Hz}, 3 \mathrm{H}), 0.87(\mathrm{~d}, J=6.8 \mathrm{~Hz}$, $3 \mathrm{H}), 0.83(\mathrm{t}, J=6.6 \mathrm{~Hz}, 3 \mathrm{H}) ;{ }^{13} \mathrm{C} \mathrm{NMR}\left(75 \mathrm{MHz}, \mathrm{CDCl}_{3}\right): \delta 200.3,137.7,132.7,132.3$, $130.2,128.5,128.2,45.3,41.8,32.6,31.8,31.7,29.4,28.7,22.6,20.6,18.9,14.1 ; \mathrm{MS}$ $m / z$ (relative intensity): $286\left(\mathrm{M}^{+}, 7\right), 243\left(\mathrm{M}^{+}-\mathrm{C}_{3} \mathrm{H}_{7}, 17\right)$; HRMS m/z Calcd. for $\mathrm{C}_{20} \mathrm{H}_{30} \mathrm{O}$ $\left(\mathrm{M}^{+}\right)$: 286.2297. Found: 286.2299; the enantiomeric purity was determined by HPLC analysis: $($ ChiralPak AD-H, hexane $/ i-\mathrm{PrOH}=99.75 / 0.25$, flow rate $=1.0 \mathrm{~mL} / \mathrm{min}), \mathrm{t}_{\mathrm{R}}=$ $8.0 \min (R), \mathrm{t}_{\mathrm{R}}=9.6 \min (S)$. 
(S,E)-Methyl 2-(2-oxo-2-phenylethyl)dec-3-enoate (3m)

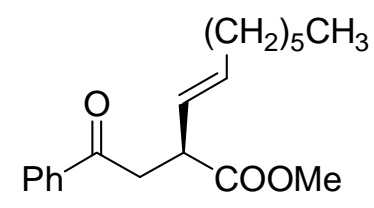

$[\alpha]^{25}{ }_{589}+68.7\left(98.4: 1.6\right.$ er, c $\left.3.37, \mathrm{CHCl}_{3}\right)$; IR (neat): 1737, 1688, 1598, 1449, 1213, 1165, 965, 756, $690 \mathrm{~cm}^{-1} ;{ }^{1} \mathrm{H}$ NMR (300 MHz, $\left.\mathrm{CDCl}_{3}\right): \delta 7.94(\mathrm{~d}, J=7.6 \mathrm{~Hz}, 2 \mathrm{H}), 7.53(\mathrm{t}, J=$ $7.0 \mathrm{~Hz}, 1 \mathrm{H}), 7.43(\mathrm{dd}, J=7.6,7.0 \mathrm{~Hz}, 2 \mathrm{H}), 5.63(\mathrm{dt}, J=15.4,6.6 \mathrm{~Hz}, 1 \mathrm{H}), 5.46(\mathrm{dd}, J=$ 15.4, $7.7 \mathrm{~Hz}, 1 \mathrm{H}), 3.72-3.48(\mathrm{~m}, 5 \mathrm{H}), 3.10(\mathrm{dd}, J=17.4,4.3 \mathrm{~Hz}), 1.99(\mathrm{q}, J=6.6 \mathrm{~Hz}, 2 \mathrm{H})$, 1.39-1.17 (m, 8H), $0.85(\mathrm{t}, J=6.6 \mathrm{~Hz}, 3 \mathrm{H}) ;{ }^{13} \mathrm{C} \mathrm{NMR}\left(75 \mathrm{MHz}, \mathrm{CDCl}_{3}\right): \delta 197.7,174.25$, 136.6, 134.4, 133.2, 128.6, 128.1, 126.0, 52.1, 43.9, 41.0, 32.5, 31.6, 29.0, 28.7, 22.6, 14.1; MS m/z (relative intensity): $302\left(\mathrm{M}^{+}, 5\right), 270\left(\mathrm{M}^{+}-\mathrm{CH}_{3} \mathrm{OH}, 24\right) ; \mathrm{HRMS} m / z$ Calcd. for $\mathrm{C}_{19} \mathrm{H}_{26} \mathrm{O}_{3}\left(\mathrm{M}^{+}\right)$: 302.1882 . Found: 302.1880 ; the enantiomeric purity was determined by HPLC analysis: $($ Chiralcel OD, hexane $/ \mathrm{i}-\mathrm{PrOH}=99 / 1$, flow rate $=1 \mathrm{~mL} / \mathrm{min}), \mathrm{t}_{\mathrm{R}}=$ $14.7 \min (R), \mathrm{t}_{\mathrm{R}}=18.1 \min (S)$.

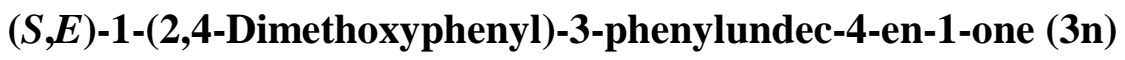

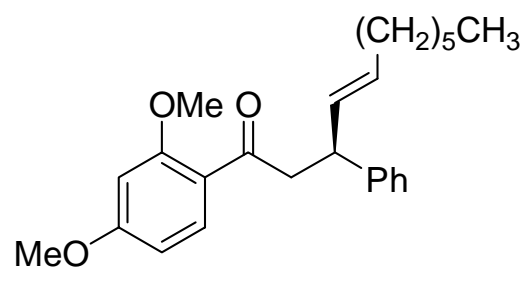

$[\alpha]_{589}^{25}+15.7$ (99.5:0.5 er, c 3.8, $\left.\mathrm{CHCl}_{3}\right)$; IR (neat): 1666, 1601, 1259, 1212, 1028, 968, 836, 752, $700 \mathrm{~cm}^{-1} ;{ }^{1} \mathrm{H}$ NMR (300 MHz, $\left.\mathrm{CDCl}_{3}\right): \delta 7.65(\mathrm{~d}, J=8.7 \mathrm{~Hz}, 1 \mathrm{H}), 7.31-7.11$ (m, 5H), $6.48(\mathrm{dd}, J=8.7,1.7 \mathrm{~Hz}, 1 \mathrm{H}), 6.43(\mathrm{~d}, J=1.7 \mathrm{~Hz}, 1 \mathrm{H}), 5.57$ (dd, $J=15.3,7.2$ Hz, 1H), 5.36 (dt, $J=15.3,6.6 \mathrm{~Hz}, 1 \mathrm{H}), 3.97$ (q, $J=7.2 \mathrm{~Hz}, 1 \mathrm{H}), 3.85(\mathrm{~s}, 3 \mathrm{H}), 3.83$ (s, $3 \mathrm{H}), 3.40-3.27(\mathrm{~m}, 2 \mathrm{H}), 1.94(\mathrm{q}, J=6.6 \mathrm{~Hz}, 2 \mathrm{H}), 1.35-1.16(\mathrm{~m}, 8 \mathrm{H}), 0.85(\mathrm{t}, J=6.6 \mathrm{~Hz}$, $3 \mathrm{H}) ;{ }^{13} \mathrm{C}$ NMR $\left(75 \mathrm{MHz}, \mathrm{CDCl}_{3}\right): \delta 199.0,164.2,160.4,144.7,132.7,132.7,130.7$, 
$128.3,127.6,126.0,121.7,105.1,98.3,55.5,55.5,49.8,44.3,32.5,31.7,29.4,28.8,22.6$, 14.1; MS $m / z$ (relative intensity): $380\left(\mathrm{M}^{+}, 9\right)$; HRMS $m / z$ Calcd. for $\mathrm{C}_{25} \mathrm{H}_{32} \mathrm{O}_{3}\left(\mathrm{M}^{+}\right)$: 380.2351. Found: 380.2361 ; the enantiomeric purity was determined by HPLC analysis: $($ ChiralPak AD-H, hexane $/ i-\mathrm{PrOH}=98 / 2$, flow rate $=1.0 \mathrm{~mL} / \mathrm{min}), \mathrm{t}_{\mathrm{R}}=15.4 \mathrm{~min}(R), \mathrm{t}_{\mathrm{R}}$ $=17.7 \min (S)$.

$(S, E)$-4-Phenyldodec-5-en-2-one (3o $)^{7}$<smiles>C/C=C\C(CC(C)=O)c1ccccc1</smiles>

$[\alpha]_{589}^{25}+1.78\left(99.1: 0.9 \mathrm{er}, \mathrm{c} 1.01, \mathrm{CHCl}_{3}\right)\left[\right.$ lit. $[\alpha]^{27}{ }_{589}-1.4\left(90: 10 \mathrm{er}, \mathrm{c} 1.04, \mathrm{CHCl}_{3}\right)$ for the $R$ enantiomer]; IR (neat): $1718,967,754,700 \mathrm{~cm}^{-1} ;{ }^{1} \mathrm{H}$ NMR (300 MHz, $\left.\mathrm{CDCl}_{3}\right): \delta$ 7.32-7.13 (m, 5H), $5.53(\mathrm{dd}, J=15.4,6.9 \mathrm{~Hz}, 1 \mathrm{H}), 5.41(\mathrm{dt}, J=15.4,6.7 \mathrm{~Hz}, 1 \mathrm{H}), 3.83$ (q, $J=6.9 \mathrm{~Hz}, 1 \mathrm{H}), 2.87-2.70(\mathrm{~m}, 2 \mathrm{H}), 2.01(\mathrm{~s}, 3 \mathrm{H}), 1.96(\mathrm{q}, J=6.7 \mathrm{~Hz}, 2 \mathrm{H}), 1.35-1.15$ $(\mathrm{m}, 8 \mathrm{H}), 0.85(\mathrm{t}, J=6.6 \mathrm{~Hz}, 3 \mathrm{H}) ;{ }^{13} \mathrm{C}$ NMR $\left(75 \mathrm{MHz}, \mathrm{CDCl}_{3}\right): \delta 207.3,143.8,132.0$, $131.1,128.5,127.4,126.4,49.8,43.9,32.4,31.6,30.6,29.3,28.8,22.6,14.0$; the enantiomeric purity was determined by HPLC analysis: (Chiralcel OD, hexane/ $i-\mathrm{PrOH}=$ 99.75/0.25, flow rate $=1 \mathrm{~mL} / \mathrm{min}), \mathrm{t}_{\mathrm{R}}=17.4 \min (R), \mathrm{t}_{\mathrm{R}}=19.1 \mathrm{~min}(S)$.

$(S, E)-1,3,5-T r i p h e n y l p e n t-4-e n-1-o n e ~(3 p)^{8}$<smiles>O=C(CC(/C=C/c1ccccc1)c1ccccc1)c1ccccc1</smiles> 
$[\alpha]_{589}^{25}-6.03$ (99.3:0.7 er, c 3.3, $\mathrm{CHCl}_{3}$ ); IR (neat): 1682, 1596, 1492, 1447, 799, 743, $691 \mathrm{~cm}^{-1} ;{ }^{1} \mathrm{H}$ NMR $\left(300 \mathrm{MHz}, \mathrm{CDCl}_{3}\right): \delta 7.98(\mathrm{~d}, J=7.4 \mathrm{~Hz}, 2 \mathrm{H}), 7.61-7.17(\mathrm{~m}, 13 \mathrm{H})$, 6.52-6.38 (m, 2H), $4.36(\mathrm{q}, J=6.5 \mathrm{~Hz}, 1 \mathrm{H}), 3.62-3.45(\mathrm{~m}, 2 \mathrm{H}) ;{ }^{13} \mathrm{C} \mathrm{NMR}(75 \mathrm{MHz}$, $\left.\mathrm{CDCl}_{3}\right): \delta 198.1,143.3,137.2,137.1,133.1,132.6,130.1,128.7,128.6,128.5,128.1$, $127.8,127.3,126.7,126.3,44.5,44.0$; the enantiomeric purity was determined by HPLC analysis: $($ ChiralPak AD-H, hexane $/ i-\mathrm{PrOH}=99.5 / 0.5$, flow rate $=1 \mathrm{~mL} / \mathrm{min}), \mathrm{t}_{\mathrm{R}}=39.3$ $\min (R), \mathrm{t}_{\mathrm{R}}=41.0 \min (S)$.

(S,E)-6-(Benzyloxy)-1,3-diphenylhex-4-en-1-one (3q)

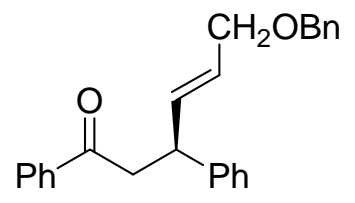

$[\alpha]_{589}^{25}-1.64$ (98.6:1.4 er, c 1.34, $\left.\mathrm{CHCl}_{3}\right)$; IR (neat): 1686, 1450, 1215, 976, 748, $691 \mathrm{~cm}^{-}$

1, ${ }^{1} \mathrm{H}$ NMR (300 MHz, $\left.\mathrm{CDCl}_{3}\right): \delta 7.93(\mathrm{~d}, J=7.3 \mathrm{~Hz}, 2 \mathrm{H}), 7.58-7.16(\mathrm{~m}, 13 \mathrm{H}), 5.96(\mathrm{dd}$, $J=15.5,7.0 \mathrm{~Hz}, 1 \mathrm{H}), 5.61(\mathrm{dt}, J=15.5,5.9 \mathrm{~Hz}, 1 \mathrm{H}), 4.50-4.40(\mathrm{~m}, 2 \mathrm{H}), 4.18(\mathrm{q}, J=7.0$ $\mathrm{Hz}, 1 \mathrm{H}), 4.04-3.91(\mathrm{~m}, 2 \mathrm{H}), 3.33-3.51(\mathrm{~m}, 2 \mathrm{H}) ;{ }^{13} \mathrm{C} \mathrm{NMR}\left(75 \mathrm{MHz}, \mathrm{CDCl}_{3}\right): \delta 198.1$, 143.2, 138.3, 137.1, 136.1, 133.1, 128.7, 128.6, 128.4, 128.1, 127.8, 127.8, 127.6, 126.9, 126.6, 72.0, 70.6, 44.3, 43.5; MS (CI, $\left.\mathrm{NH}_{3}\right) \mathrm{m} / z$ (relative intensity): $374\left(\mathrm{M} \mathrm{NH}_{4}{ }^{+}, 100\right)$, 249 (18); HRMS (ESI + LiOAc) $m / z$ Calcd. for $\mathrm{C}_{25} \mathrm{H}_{24} \mathrm{LiO}_{2}\left(\mathrm{M}+\mathrm{Li}^{+}\right): 363.1936$. Found: 363.1946; the enantiomeric purity was determined by HPLC analysis: (ChiralCel OD-H, hexane $/ i-\mathrm{PrOH}=96.75 / 3.25$, flow rate $=1.0 \mathrm{~mL} / \mathrm{min}), \mathrm{t}_{\mathrm{R}}=25.9 \mathrm{~min}(S), \mathrm{t}_{\mathrm{R}}=36.8 \mathrm{~min}$ $(R)$ 


\section{(S,Z)-1,3-Diphenylundec-4-en-1-one (3r)}<smiles>C/C=C\C(CC(=O)c1ccccc1)c1ccccc1</smiles>

$[\alpha]_{589}^{25}+68.5$ (95.5:4.5 er, c 2.2, $\left.\mathrm{CHCl}_{3}\right)$; IR (neat): $1687,1598,1449,747,698 \mathrm{~cm}^{-1} ;{ }^{1} \mathrm{H}$ NMR $\left(300 \mathrm{MHz}, \mathrm{CDCl}_{3}\right): \delta 7.93(\mathrm{~d}, J=7.5 \mathrm{~Hz}, 2 \mathrm{H}), 7.54(\mathrm{~d}, J=7.3 \mathrm{~Hz}, 1 \mathrm{H}), 7.44$ (dd, $J$ $=7.5,7.3 \mathrm{~Hz}, 2 \mathrm{H}), 7.32-7.13(\mathrm{~m}, 5 \mathrm{H}), 5.56(\mathrm{dd}, J=10.6,9.8 \mathrm{~Hz}, 1 \mathrm{H}), 5.42(\mathrm{dt}, J=10.6$, $7.2 \mathrm{~Hz}, 1 \mathrm{H}), 4.40(\mathrm{ddd}, J=9.8,7.9,6.3 \mathrm{~Hz}, 1 \mathrm{H}), 3.41(\mathrm{dd}, J=16.1,6.3 \mathrm{~Hz}, 1 \mathrm{H}), 3.29$ (dd, $J=16.1,7.9 \mathrm{~Hz}, 1 \mathrm{H}), 2.10(\mathrm{q}, J=7.2 \mathrm{~Hz}, 2 \mathrm{H}), 1.35-1.14(\mathrm{~m}, 8 \mathrm{H}), 0.86(\mathrm{t}, J=6.3 \mathrm{~Hz}$, $3 \mathrm{H}) ;{ }^{13} \mathrm{C} \mathrm{NMR}\left(75 \mathrm{MHz}, \mathrm{CDCl}_{3}\right): \delta 198.5,144.6,137.1,133.0,131.7,131.0,128.6$, 128.6, 128.1, 127.2, 126.3, 45.7, 39.2, 31.7, 29.5, 29.0, 27.6, 22.6, 14.1; MS $m / z$ (relative intensity): $380\left(\mathrm{M}^{+}, 9\right)$; HRMS $m / z$ Calcd. for $\mathrm{C}_{23} \mathrm{H}_{28} \mathrm{O}\left(\mathrm{M}^{+}\right)$: 320.2140. Found: 320.2132; the enantiomeric purity was determined by HPLC analysis: (ChiralPak AD-H, hexane/ $i$ $\operatorname{PrOH}=99.5 / 0.5$, flow rate $=1.0 \mathrm{~mL} / \mathrm{min}), \mathrm{t}_{\mathrm{R}}=9.7 \mathrm{~min}(R), \mathrm{t}_{\mathrm{R}}=11.3 \mathrm{~min}(S)$.

\section{$(S, Z)-5-B r o m o-1,3-d i p h e n y l n o n-4-e n-1-o n e ~(3 s)^{9}$}

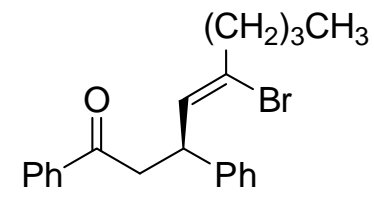

$[\alpha]_{589}^{25}+61.8\left(91: 9 \mathrm{er}, \mathrm{c} 1.63, \mathrm{CHCl}_{3}\right)$; IR (neat): 1688, 1597, 1448, 748, $698 \mathrm{~cm}^{-1} ;{ }^{1} \mathrm{H}$ NMR (300 MHz, $\left.\mathrm{CDCl}_{3}\right): \delta 7.92(\mathrm{~d}, J=7.7 \mathrm{~Hz}, 2 \mathrm{H}), 7.54(\mathrm{~d}, J=7.2 \mathrm{~Hz}, 1 \mathrm{H}), 7.43(\mathrm{dd}, J$ = 7.7, 7.2 Hz, 2H), 7.33-7.14 (m, 5H), $5.86(\mathrm{~d}, J=9.1 \mathrm{~Hz}, 1 \mathrm{H}), 4.49(\mathrm{dt}, J=9.1,7.2 \mathrm{~Hz}$, 1H), $3.45(\mathrm{dd}, J=16.1,7.2 \mathrm{~Hz}, 1 \mathrm{H}), 3.33(\mathrm{dd}, J=16.1,7.2 \mathrm{~Hz}, 1 \mathrm{H}), 2.39(\mathrm{t}, J=7.3 \mathrm{~Hz}$, 2H), $1.47(\mathrm{~m}, 2 \mathrm{H}), 1.24(\mathrm{~m}, 2 \mathrm{H}), 0.86(\mathrm{t}, J=7.2 \mathrm{~Hz}, 3 \mathrm{H}) ;{ }^{13} \mathrm{C} \mathrm{NMR}\left(75 \mathrm{MHz}, \mathrm{CDCl}_{3}\right): \delta$ $197.9,142.7,137.0,133.0,130.6,129.2,128.6,128.6,128.2,127.4,126.6,44.5,43.5$, 
41.3, 30.2, 21.5, 13.8; the enantiomeric purity was determined by HPLC analysis: $($ ChiralPak AD-H, hexane $/ i-\mathrm{PrOH}=99.5 / 0.5$, flow rate $=1.0 \mathrm{~mL} / \mathrm{min}), \mathrm{t}_{\mathrm{R}}=17.3 \min (R)$, $\mathrm{t}_{\mathrm{R}}=20.4 \min (S)$.

(S)-5-Butyl-1,3-diphenylnon-4-en-1-one (3t)<smiles>O=C(CC(C=C(Br)Br)c1ccccc1)c1ccccc1</smiles>

$[\alpha]^{25}{ }_{589}+45.2\left(96.3: 3.7 \mathrm{er}, \mathrm{c} 2.2, \mathrm{CHCl}_{3}\right) ; \mathrm{IR}$ (neat): $1688,1448,748,698 \mathrm{~cm}^{-1} ;{ }^{1} \mathrm{H} \mathrm{NMR}$ $\left(300 \mathrm{MHz}, \mathrm{CDCl}_{3}\right): \delta 7.92(\mathrm{~d}, J=7.6 \mathrm{~Hz}, 2 \mathrm{H}), 7.53(\mathrm{t}, J=7.2,1.3 \mathrm{~Hz}, 1 \mathrm{H}), 7.43(\mathrm{dd}, J=$ 7.6, 7.2 Hz, 2H), 7.32-7.14 (m, 5H), $5.33(\mathrm{~d}, J=9.7 \mathrm{~Hz}, 1 \mathrm{H}), 4.31$ (ddd, $J=9.7,6.4,7.8$ Hz, 1H), 3.37 (dd, $J=15.7,6.4 \mathrm{~Hz}, 1 \mathrm{H}), 3.26(\mathrm{dd}, \mathrm{J}=15.7,7.8 \mathrm{~Hz}, 1 \mathrm{H}), 2.16-1.88(\mathrm{~m}$, $4 \mathrm{H}), 1.41-1.14(\mathrm{~m}, 8 \mathrm{H}), 0.85(\mathrm{t}, J=6.8 \mathrm{~Hz}, 6 \mathrm{H}) ;{ }^{13} \mathrm{C} \mathrm{NMR}\left(75 \mathrm{MHz}, \mathrm{CDCl}_{3}\right): \delta 198.8$, 145.3, 141.0, 137.3, 132.9, 128.5, 128.2, 127.3, 126.7, 126.1, 46.4, 39.8, 36.5, 30.5, 30.29, 30.1, 23.0, 22.4, 14.0; MS (EI) m/z (relative intensity): 348 ( $\left.\mathbf{M}^{+}, 20\right), 291$ (29), 228 (100); HRMS (EI) $\mathrm{m} / z$ Calcd. for $\mathrm{C}_{25} \mathrm{H}_{32} \mathrm{O}\left(\mathrm{M}^{+}\right)$: 348.2453. Found: 348.2440; the enantiomeric purity was determined by HPLC analysis: (ChiralPak OD, hexane $/ i-\mathrm{PrOH}=99.75 / 0.25$, flow rate $=1.0 \mathrm{~mL} / \mathrm{min}), \mathrm{t}_{\mathrm{R}}=13.3 \min (R), \mathrm{t}_{\mathrm{R}}=15.2 \min (S)$.

(R,Z)-4-Bromo-1,3-diphenylnon-4-en-1-one (3u)<smiles>C/C=C(/Br)[C@H](CC(=O)c1ccccc1)c1ccccc1</smiles>

$[\alpha]^{25}{ }_{589}+36.0\left(95: 5 \mathrm{er}, \mathrm{c} 1.04, \mathrm{CHCl}_{3}\right)$; IR (neat): $1688,1598,1448,748,698,690 \mathrm{~cm}^{-1}$;

${ }^{1} \mathrm{H} \mathrm{NMR}\left(300 \mathrm{MHz}, \mathrm{CDCl}_{3}\right): \delta 7.96(\mathrm{dd}, J=7.5,1.3 \mathrm{~Hz}, 2 \mathrm{H}), 7.55(\mathrm{dt}, J=7.3,1.3 \mathrm{~Hz}$, 
1H), $7.45(\mathrm{dd}, J=7.5,7.3 \mathrm{~Hz}, 2 \mathrm{H}), 7.33-7.20(\mathrm{~m}, 5 \mathrm{H}), 5.89(\mathrm{t}, J=6.8 \mathrm{~Hz}, 1 \mathrm{H}), 4.48(\mathrm{dd}$, $J=7.9,6.8 \mathrm{~Hz}, 1 \mathrm{H}), 3.72(\mathrm{dd}, J=16.9,6.8 \mathrm{~Hz}, 1 \mathrm{H}), 3.45(\mathrm{dd}, J=16.9,7.9 \mathrm{~Hz}, 1 \mathrm{H}), 2.14$ $(\mathrm{m}, 2 \mathrm{H}), 1.41-1.19(\mathrm{~m}, 4 \mathrm{H}), 0.86(\mathrm{t}, J=7.0 \mathrm{~Hz}, 3 \mathrm{H}) ;{ }^{13} \mathrm{C}$ NMR $\left(75 \mathrm{MHz}, \mathrm{CDCl}_{3}\right): \delta$ 197.6, 141.1, 137.1, 133.2, 130.7, 130.4, 128.6, 128.4, 128.1, 127.8, 127.0, 50.4, 42.4, 31.0, 30.5, 22.2, 13.9; MS (CI, NH$\left.)_{3}\right) \mathrm{m} / z$ (relative intensity): $388\left(\mathrm{M}^{+}+\mathrm{NH}_{4}, 100\right), 372$ $\left(\mathrm{M}^{+}+\mathrm{H}, 23\right), 291\left(\mathrm{M}^{+}-\mathrm{Br}, 26\right)$; HRMS (EI) $\mathrm{m} / z$ Calcd. for $\mathrm{C}_{21} \mathrm{H}_{23} \mathrm{O}\left(\mathrm{M}^{+}-\mathrm{Br}\right)$ : 291.1743. Found: 291.1750; the enantiomeric purity was determined by HPLC analysis: (ChiralPak $\mathrm{AD}-\mathrm{H}$, hexane $/ \mathrm{i}-\mathrm{PrOH}=99.5 / 0.5$, flow rate $=1.0 \mathrm{~mL} / \mathrm{min}), \mathrm{t}_{\mathrm{R}}=14.1 \mathrm{~min}(S), \mathrm{t}_{\mathrm{R}}=16.9$ $\min (R)$.

$(S, E)-4-E t h y l-1,3-d i p h e n y l h e p t-4-e n-1-o n e ~(3 v){ }^{10}$

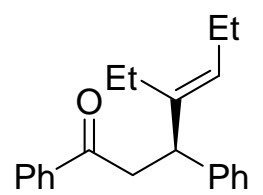

$[\alpha]^{25}{ }_{589}-37.7$ (65:35 er, c 2.57, $\left.\mathrm{CHCl}_{3}\right)$; IR (neat): 1688, 1598, 1448, 1260, 749, 700, 691 $\mathrm{cm}^{-1} ;{ }^{1} \mathrm{H}$ NMR $\left(300 \mathrm{MHz}, \mathrm{CDCl}_{3}\right): \delta 7.89(\mathrm{~d}, J=7.6 \mathrm{~Hz}, 2 \mathrm{H}), 7.52(\mathrm{t}, J=7.2 \mathrm{~Hz}, 1 \mathrm{H})$, $7.41(\mathrm{dd}, J=7.6,7.2 \mathrm{~Hz}, 2 \mathrm{H}), 7.31-7.10(\mathrm{~m}, 5 \mathrm{H}), 5.26(\mathrm{t}, J=7.0 \mathrm{~Hz}, 1 \mathrm{H}), 4.09(\mathrm{dd}, J=$ 7.3, 7.4 Hz, 1H), $3.50(\mathrm{dd}, J=16.4,7.4 \mathrm{~Hz}, 1 \mathrm{H}), 3.29(\mathrm{dd}, J=16.4,7.3 \mathrm{~Hz}, 1 \mathrm{H}), 2.18-$ $1.97(\mathrm{~m}, 3 \mathrm{H}), 1.76(\mathrm{~m}, 1 \mathrm{H}), 0.94(\mathrm{t}, J=7.6 \mathrm{~Hz}, 3 \mathrm{H}), 0.88(\mathrm{t}, J=7.6 \mathrm{~Hz}, 3 \mathrm{H}) ;{ }^{13} \mathrm{C} \mathrm{NMR}$ $\left(75 \mathrm{MHz}, \mathrm{CDCl}_{3}\right): \delta 198.9,143.7,141.9,137.5,132.8,128.5,128.3,128.1,128.0,126.3$, $126.3,46.4,43.5,23.5,21.0,14.6,13.5$; the enantiomeric purity was determined by HPLC analysis: (ChiralPak AD-H, hexane $/ i-\mathrm{PrOH}=99.5 / 0.5$, flow rate $=1.0 \mathrm{~mL} / \mathrm{min}), \mathrm{t}_{\mathrm{R}}$ $=8.8 \min (R), \mathrm{t}_{\mathrm{R}}=13.3 \min (S)$. 


\section{References}

(1) Wu, T. R.; Shen, L. X.; Chong, J. M. Org. Lett. 2004, 6, 2701-2704.

(2) Batey, R. A.; Thadani, A. N.; Lough, A. J. J. Am. Chem. Soc. 1999, 121, 450-451.

(3) Hall, D. G. Boronic Acids: Preparation and Applications in Organic Synthesis and Medicine, John Wiley \& Sons, 2005

(4) Hara, S.; Hyuga, S.; Aoyama, M.; Sato, M.; Suzuki, A. Tetrahedron Lett. 1990, 31 , 247-250.

(5) Josyula, K. V. B.; Gao, P.; Hewitt, C. Tetrahedron Lett. 2003, 44, 7789-7792.

(6) Srebnik, M.; Bhat, N. G.; Brown, H. C. Tetrahedron Lett. 1988, 29, 2635-2638.

(7) Oi, S.; Sato, T.; Inoue, Y. Tetrahedron Lett. 2004, 45, 5051-5055.

(8) Oi, S.; Honma, Y.; Inoue, Y. Org. Lett. 2002, 4, 667-669.

(9) Satoh, Y.; Serizawa, H.; Hara, S.; Suzuki, A. J. Am. Chem. Soc. 1985, 107, 52255228.

(10) El-Batta, A.; Hage, T. R.; Plotkin, S.; Bergdahl, M. Org. Lett. 2004, 6, 107-110. 


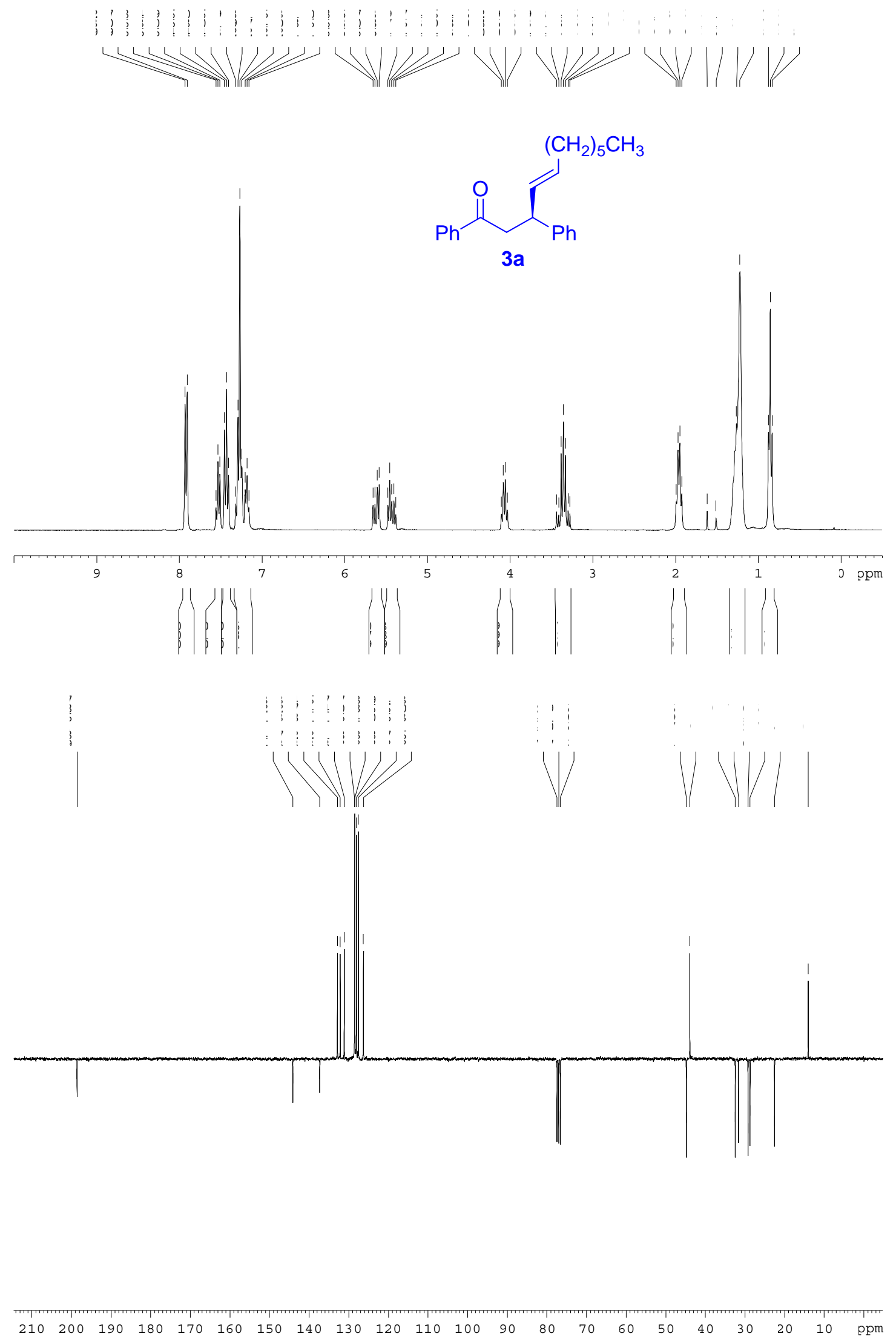




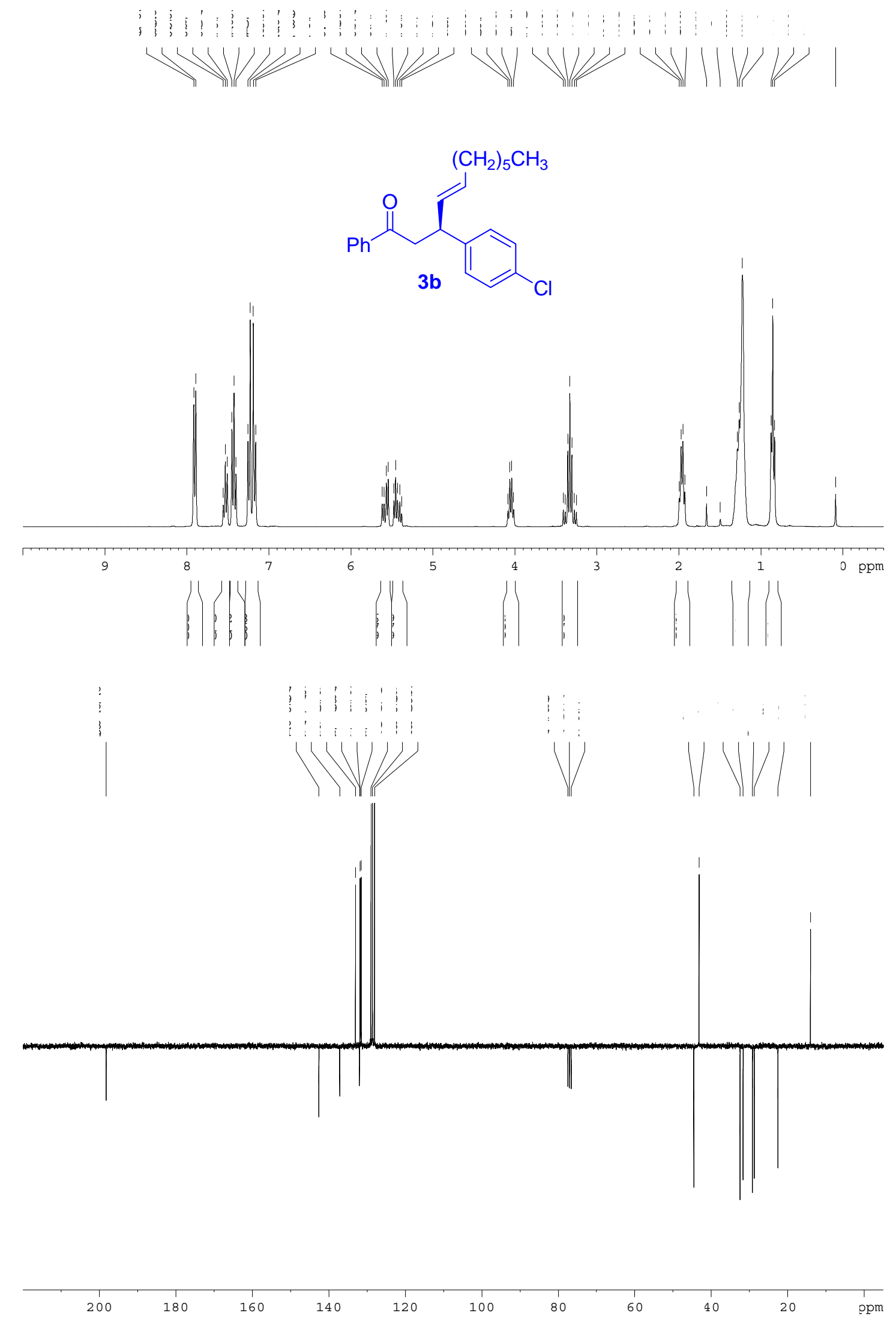




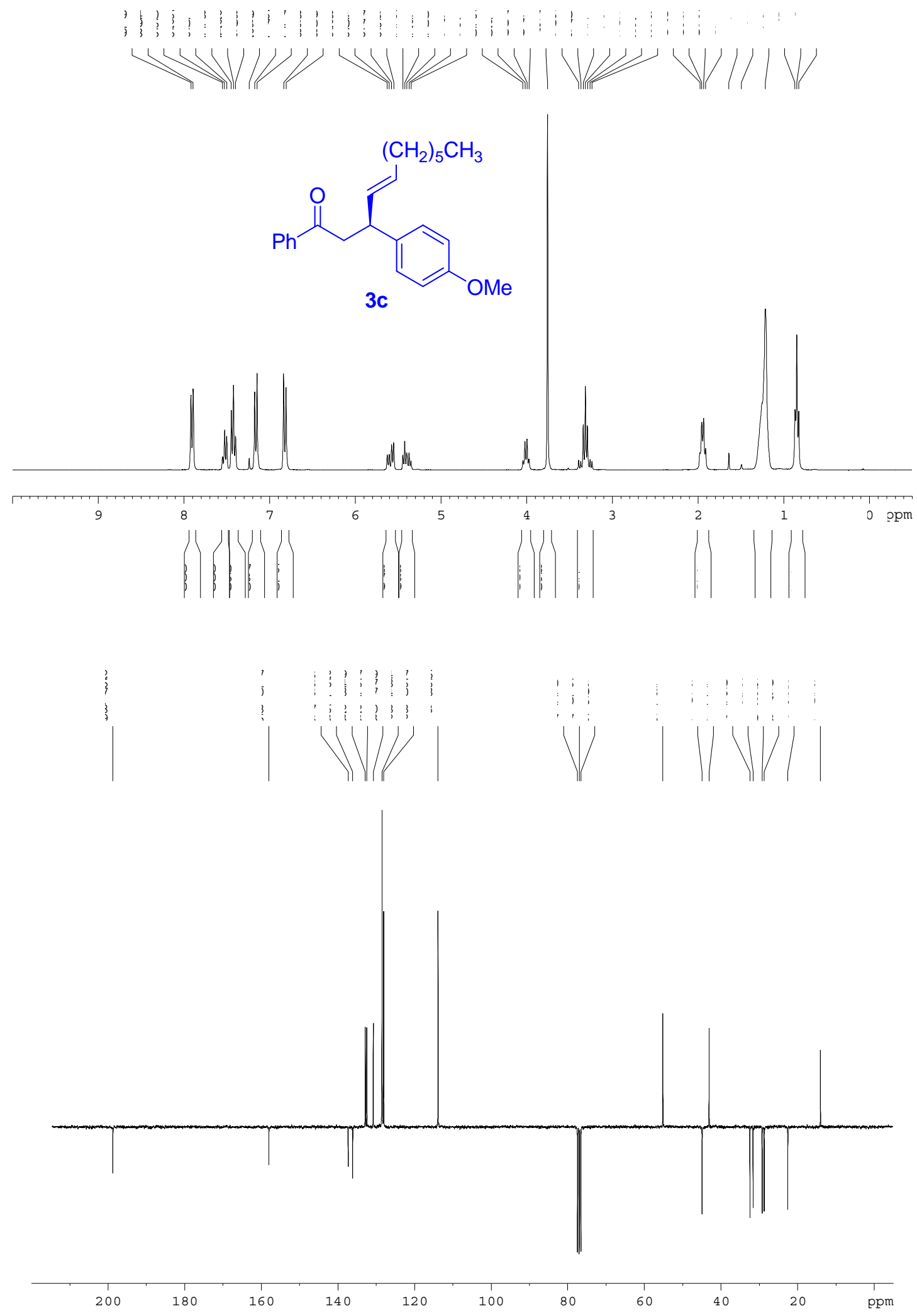




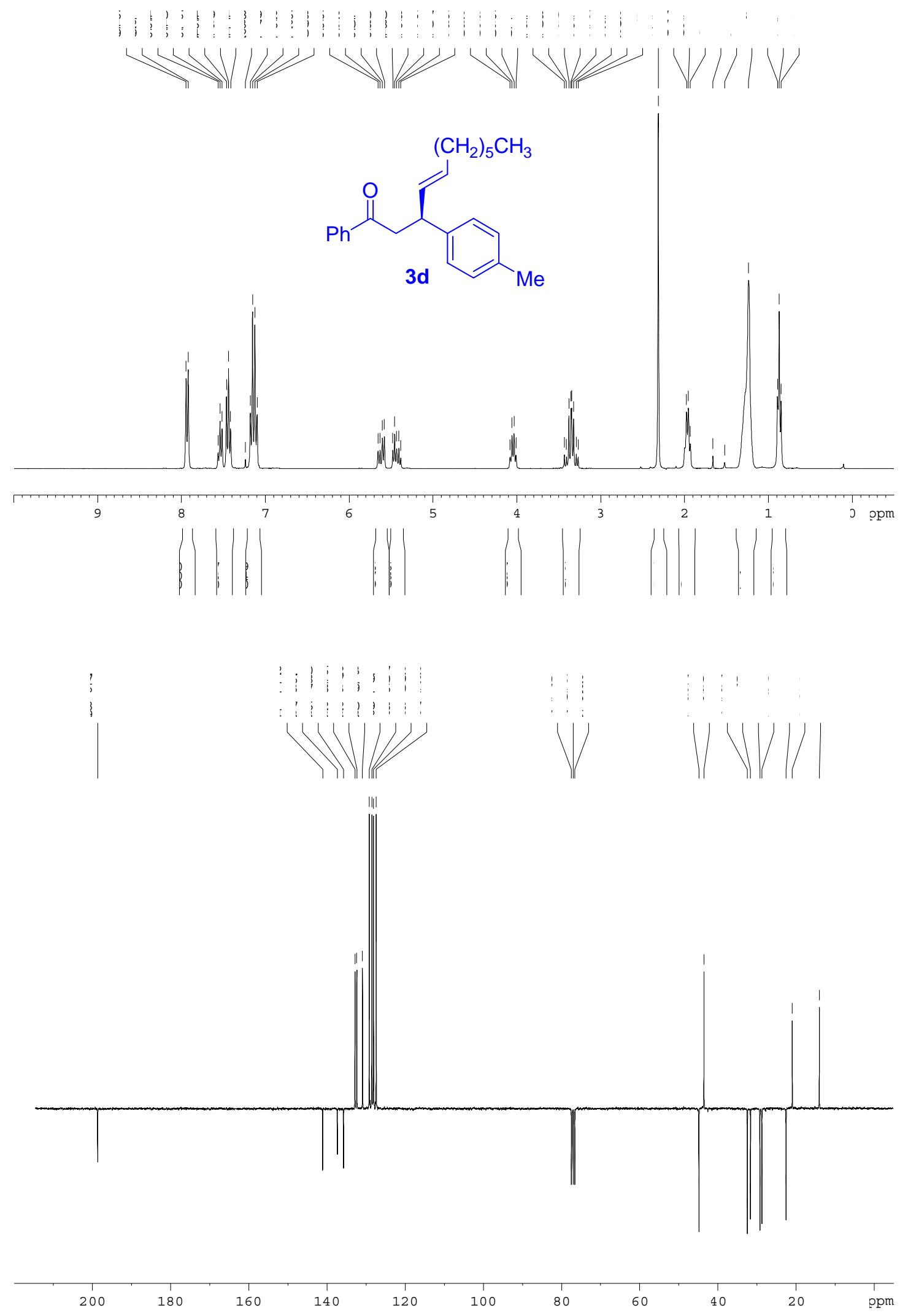




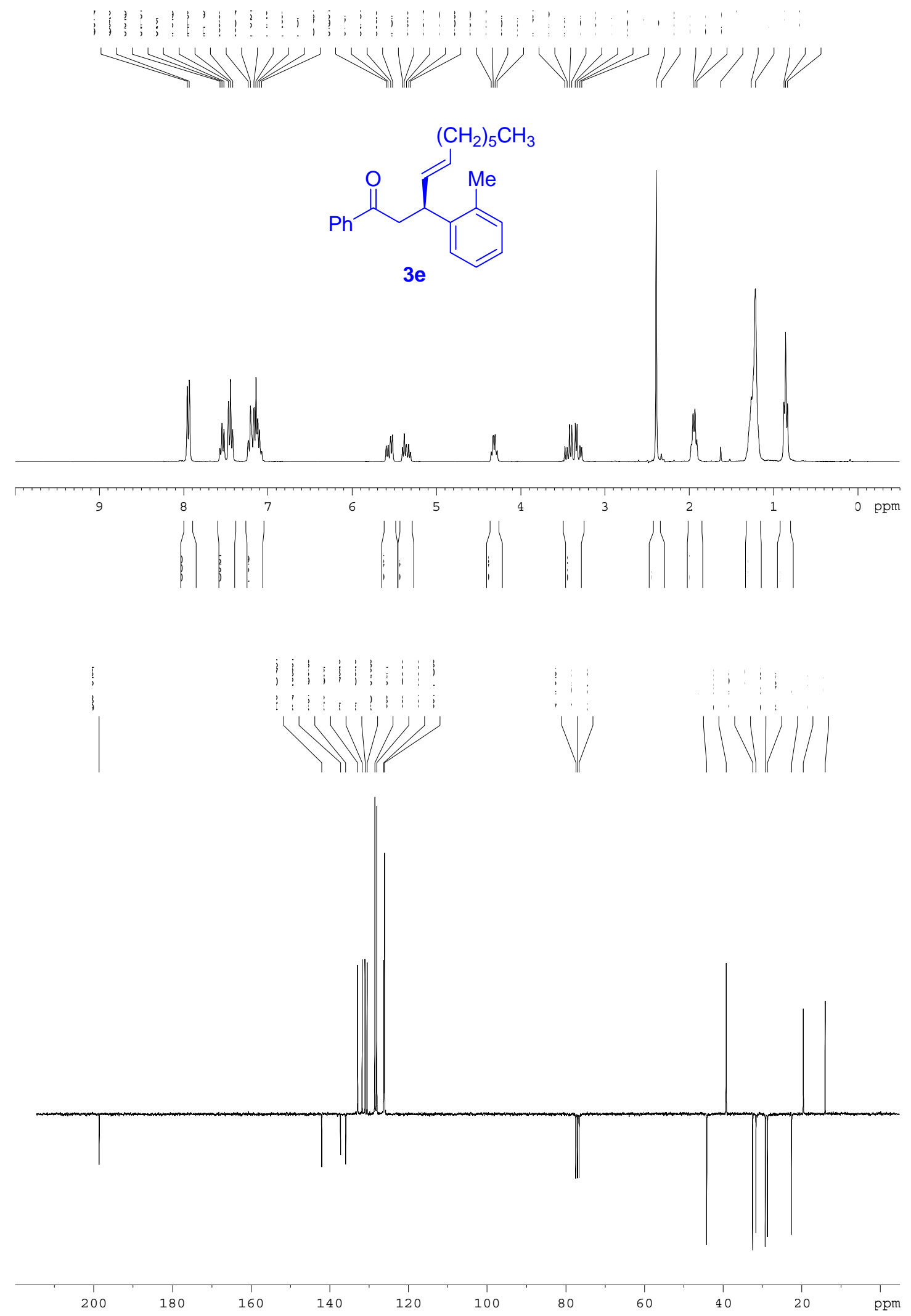




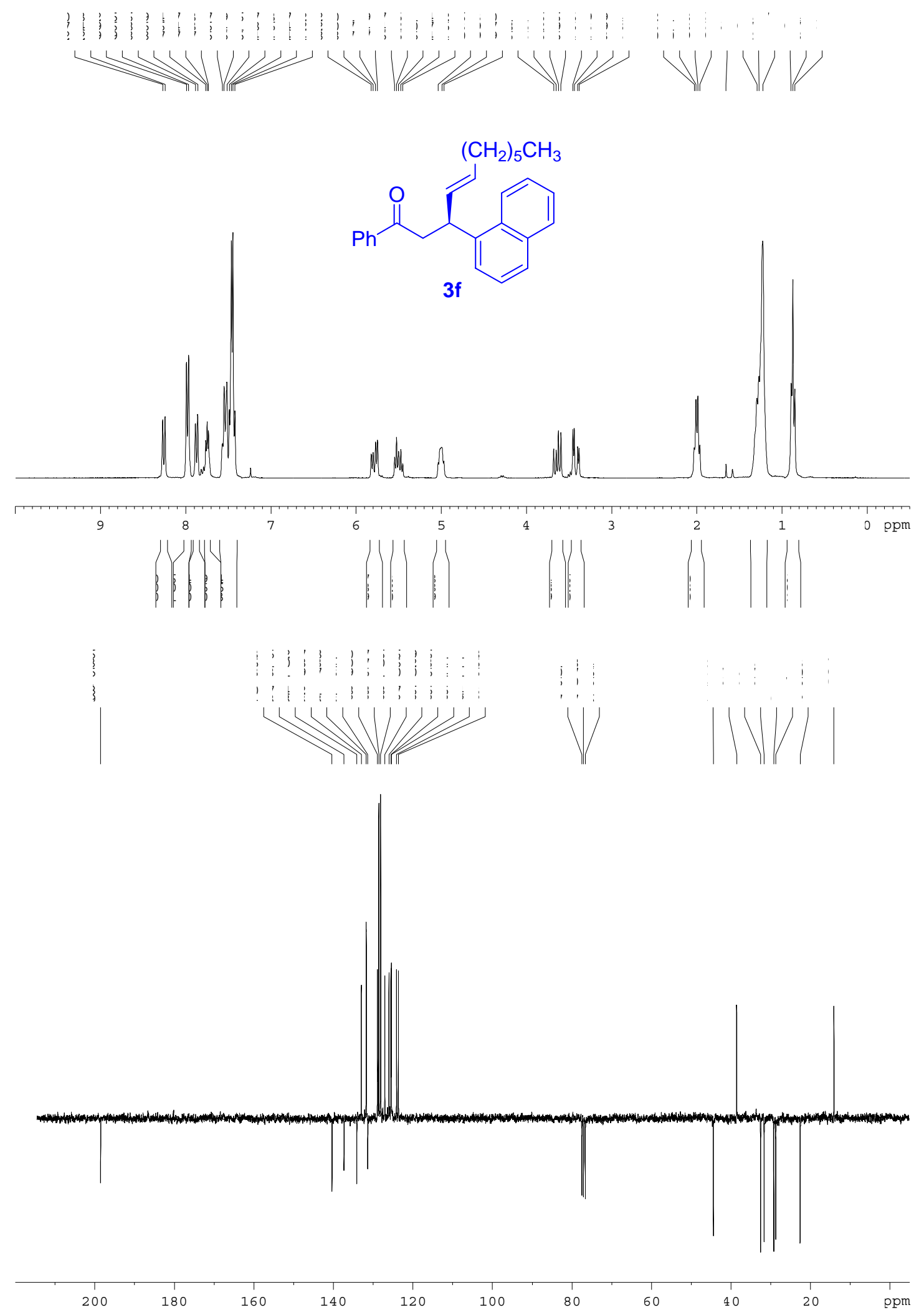




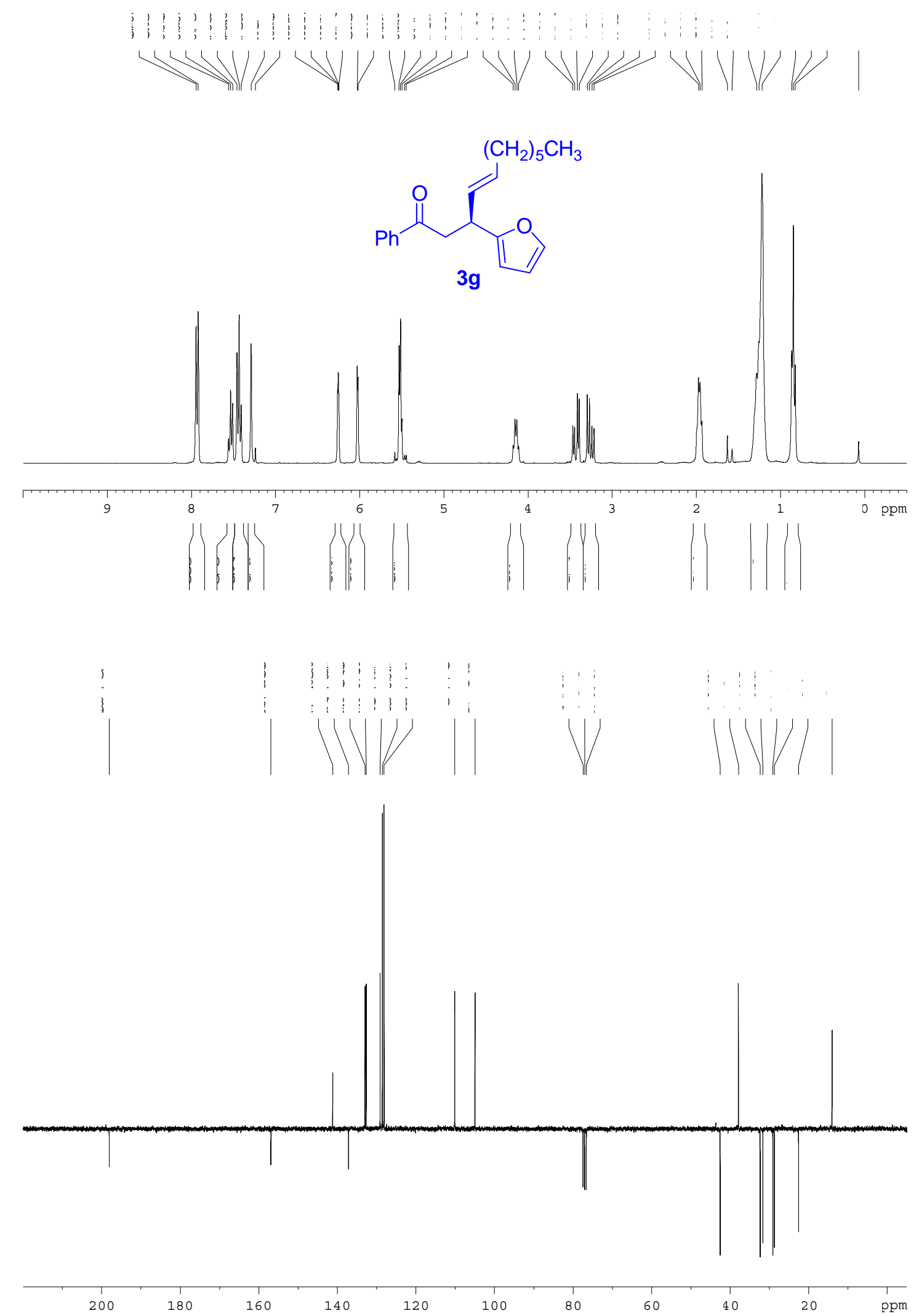




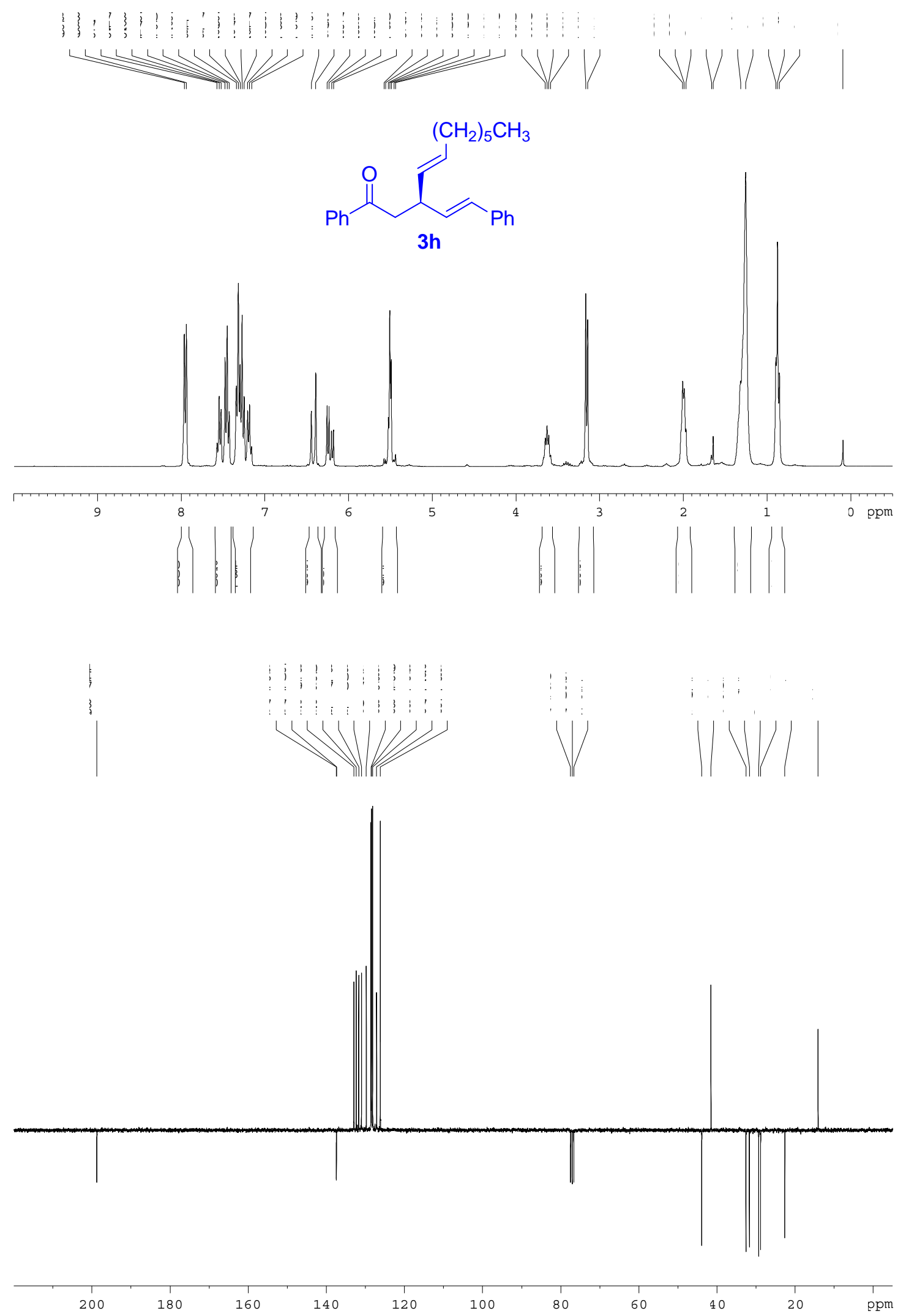




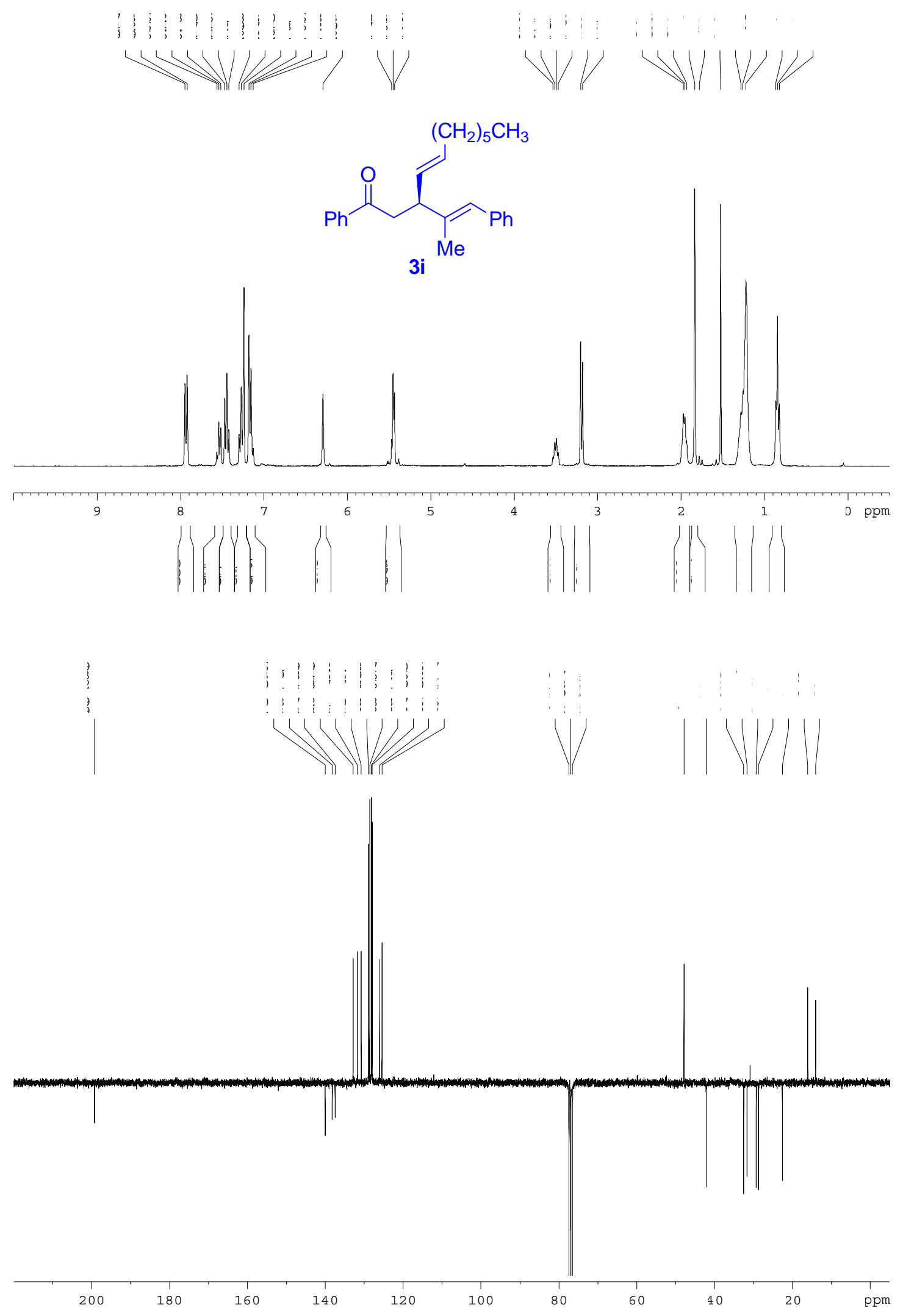




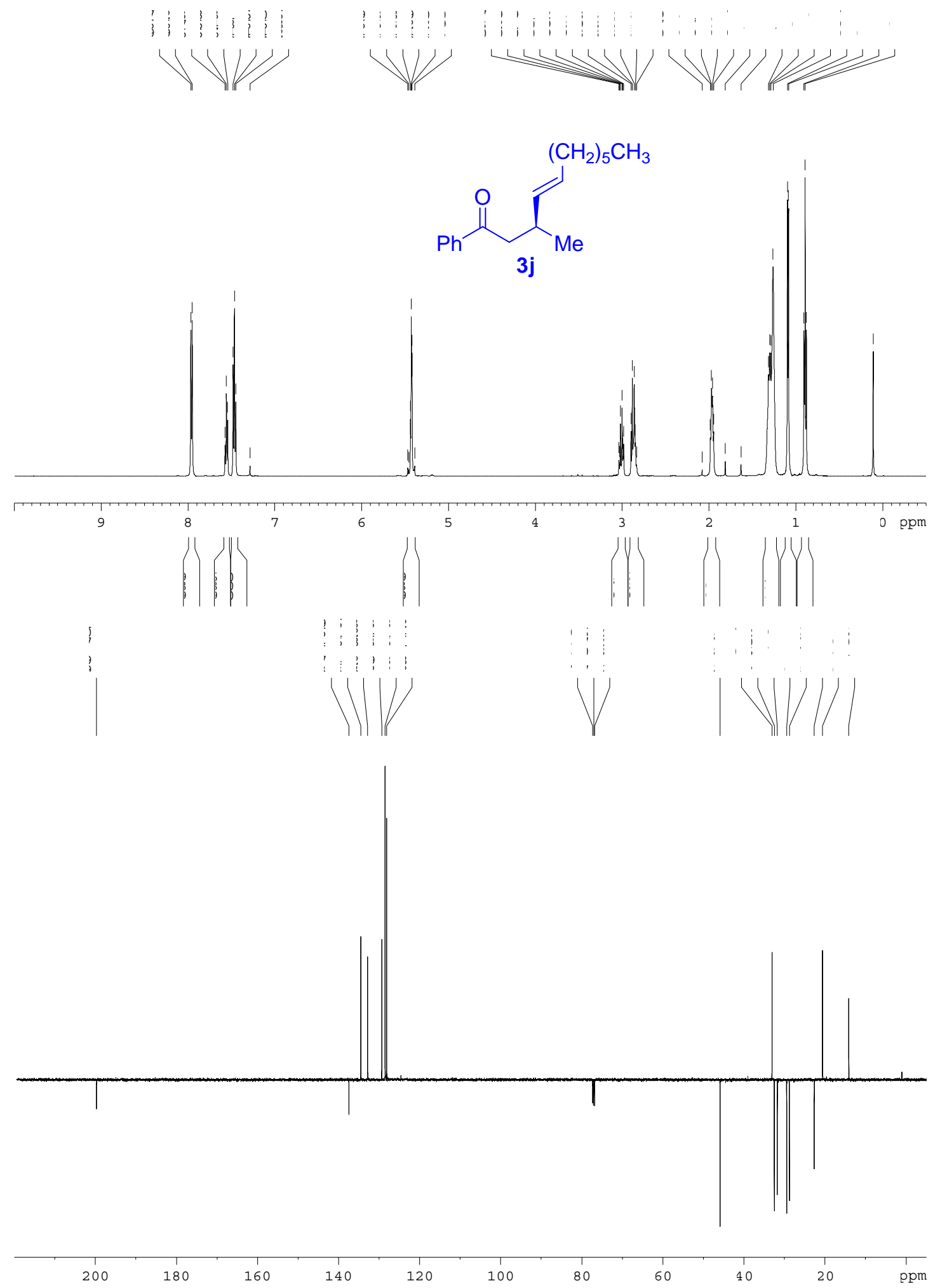




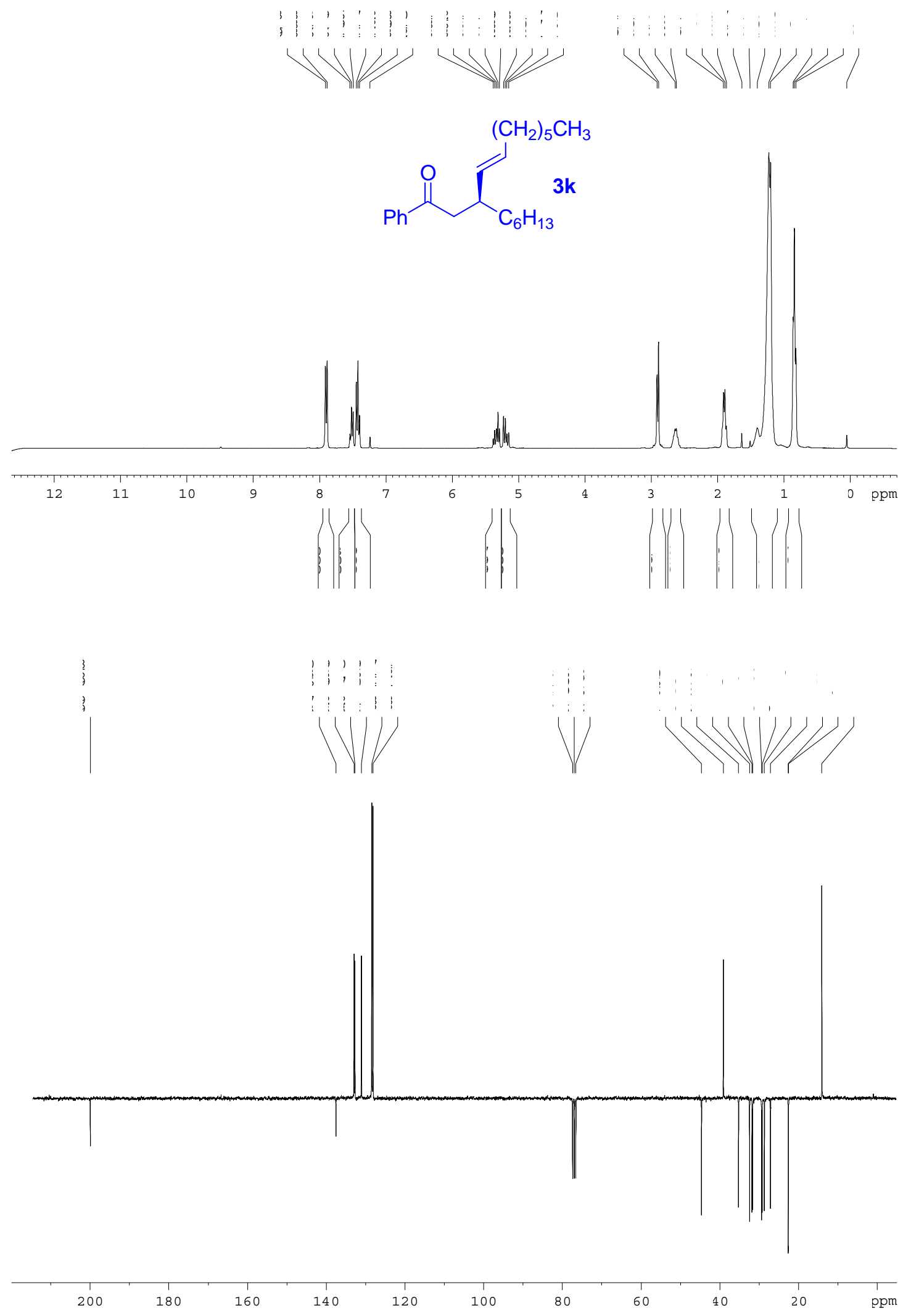




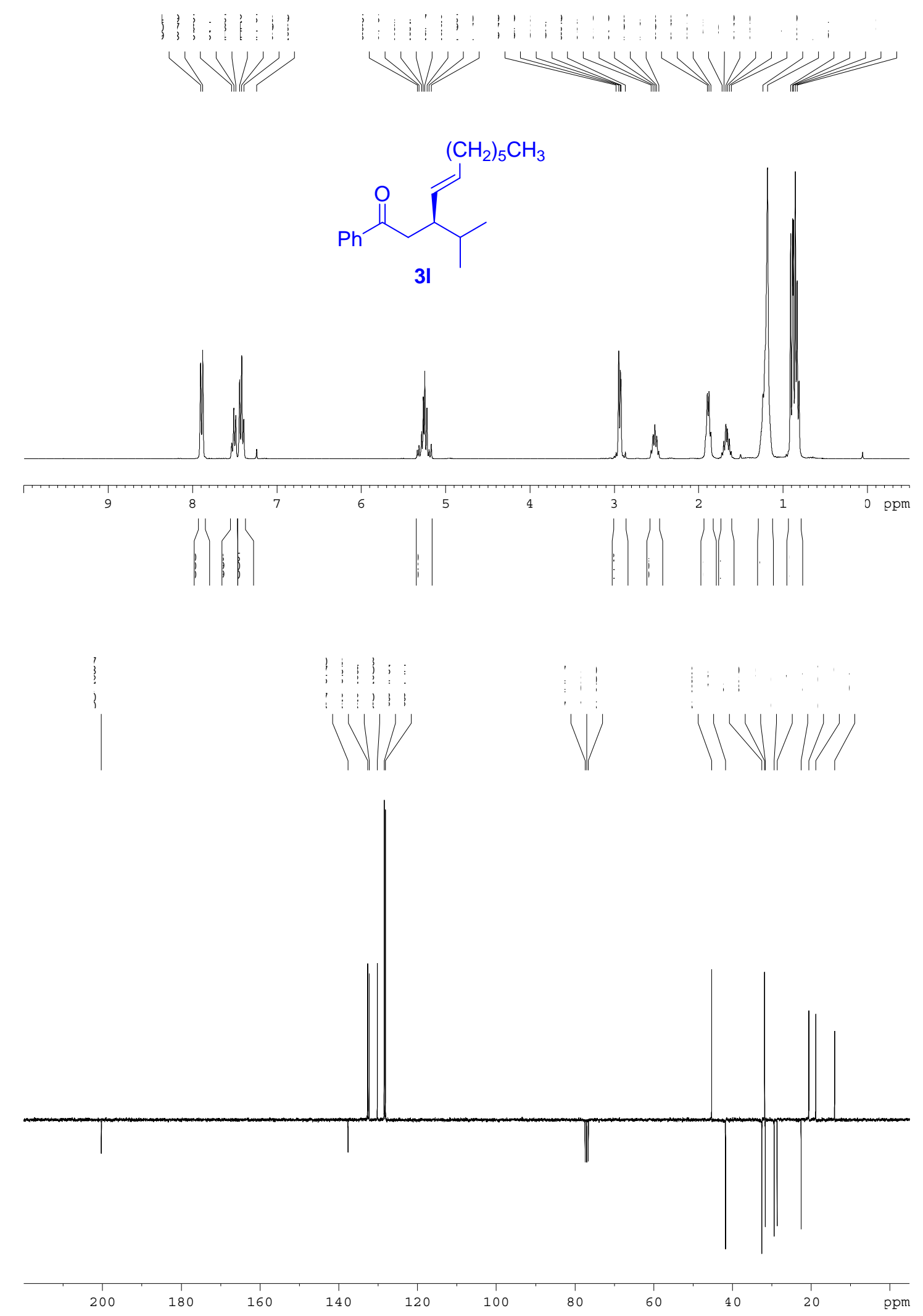




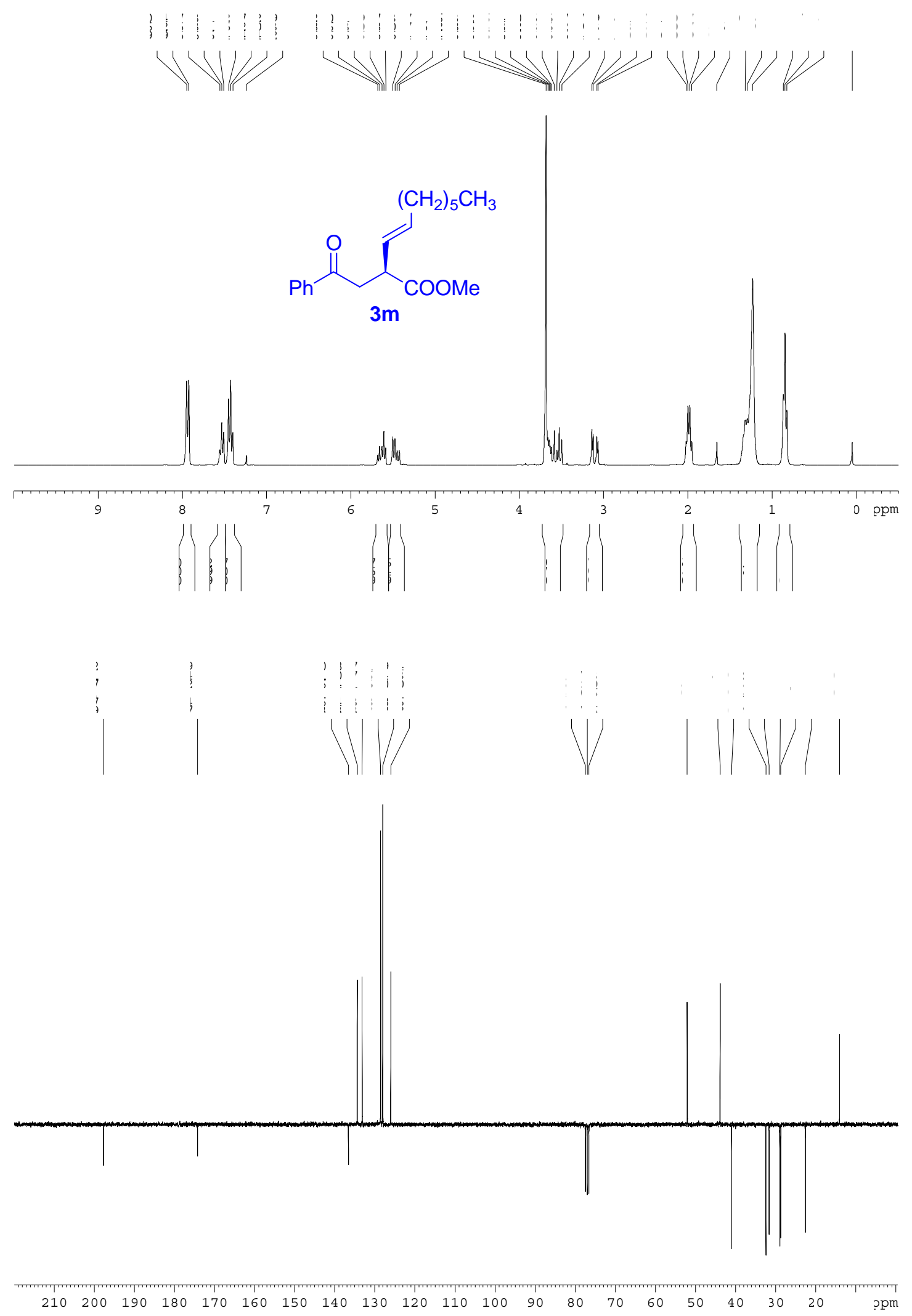



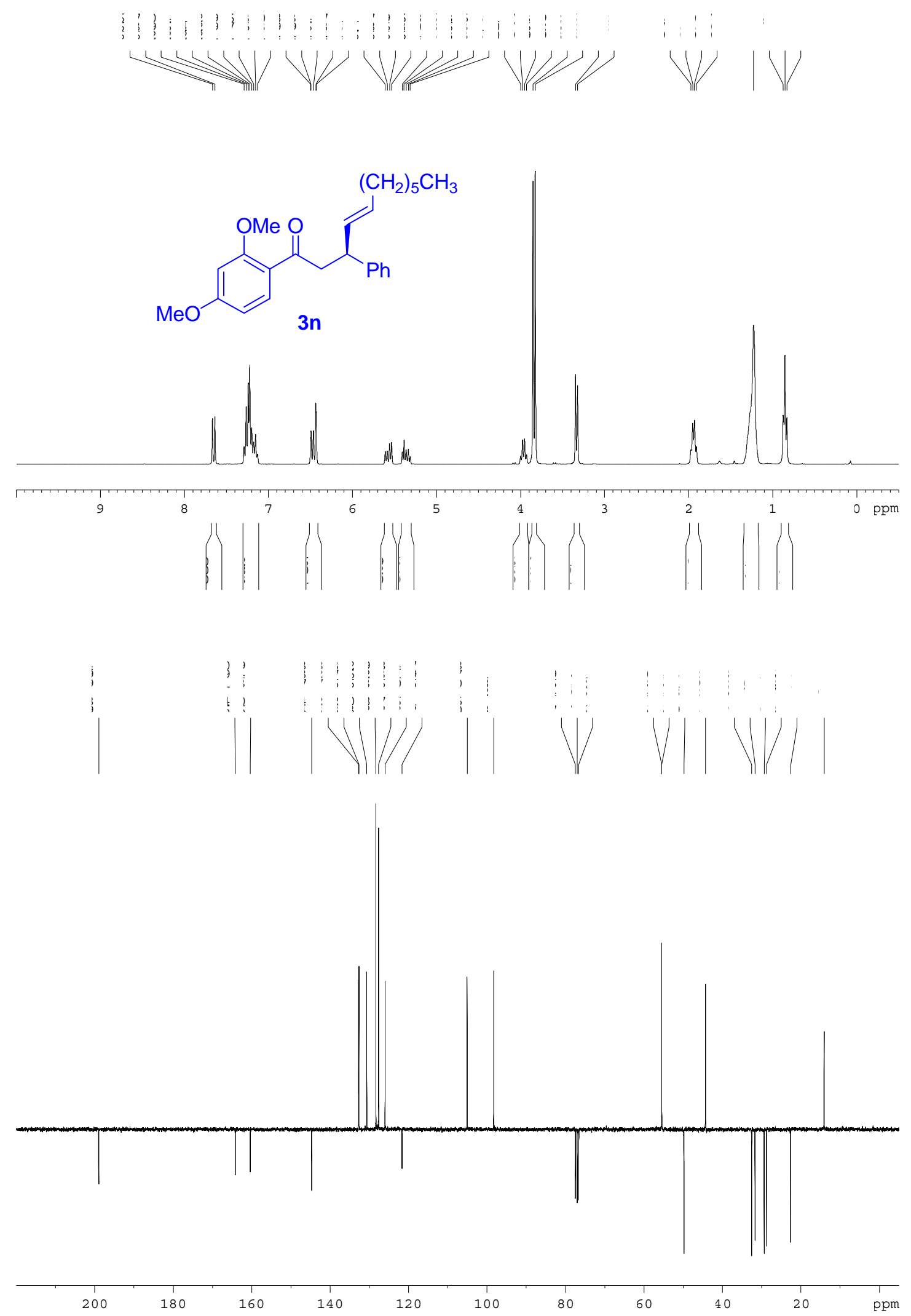


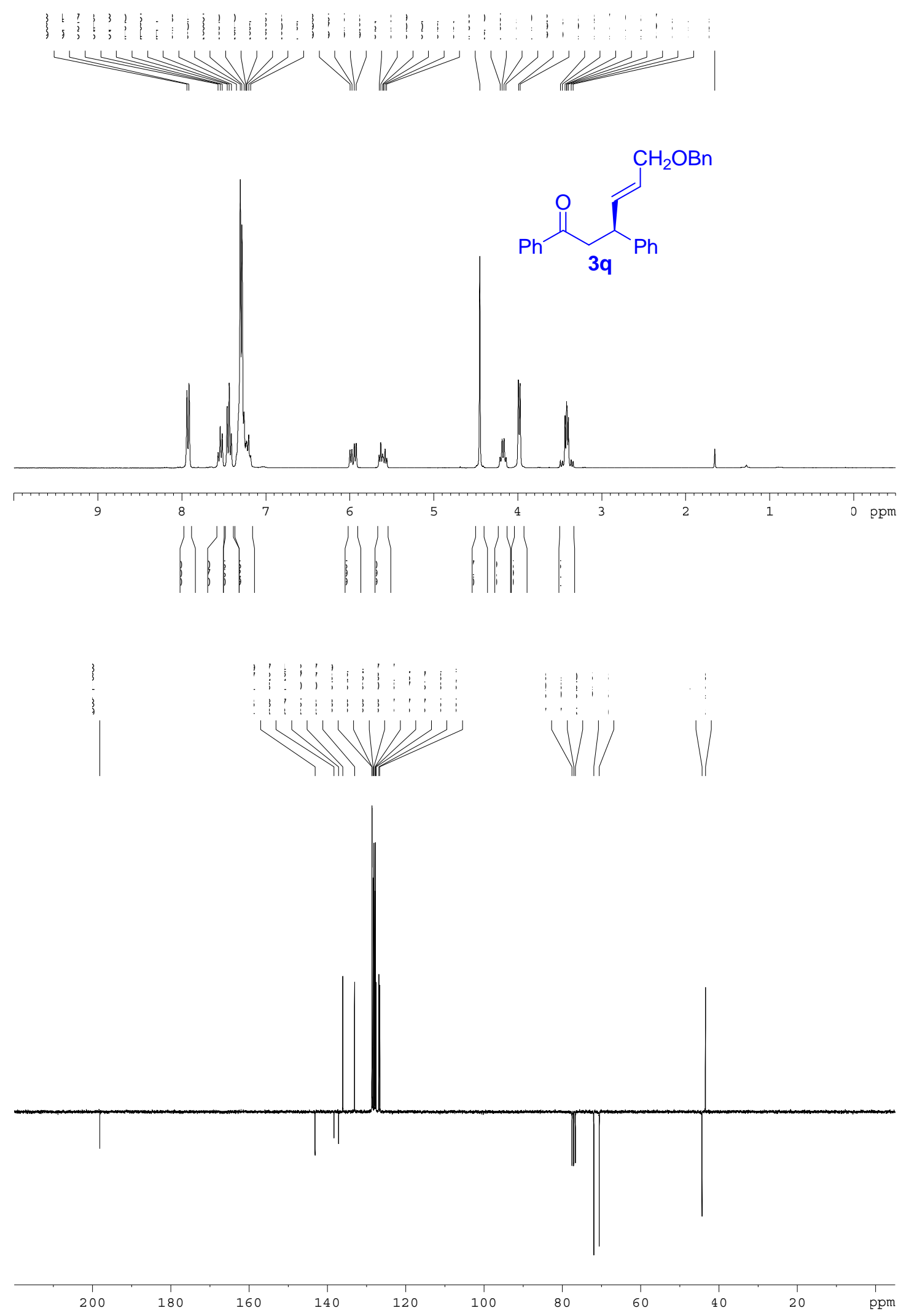




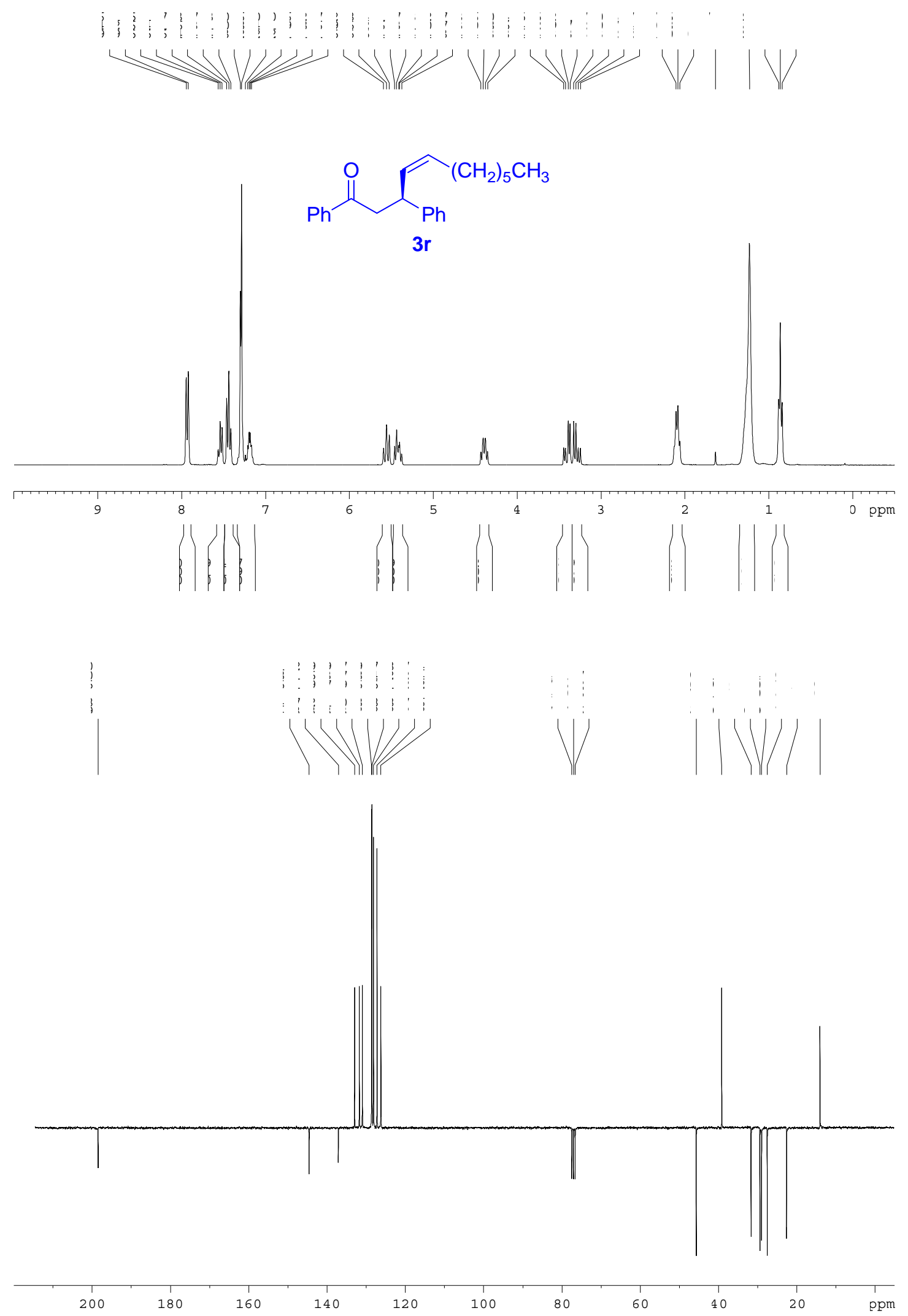




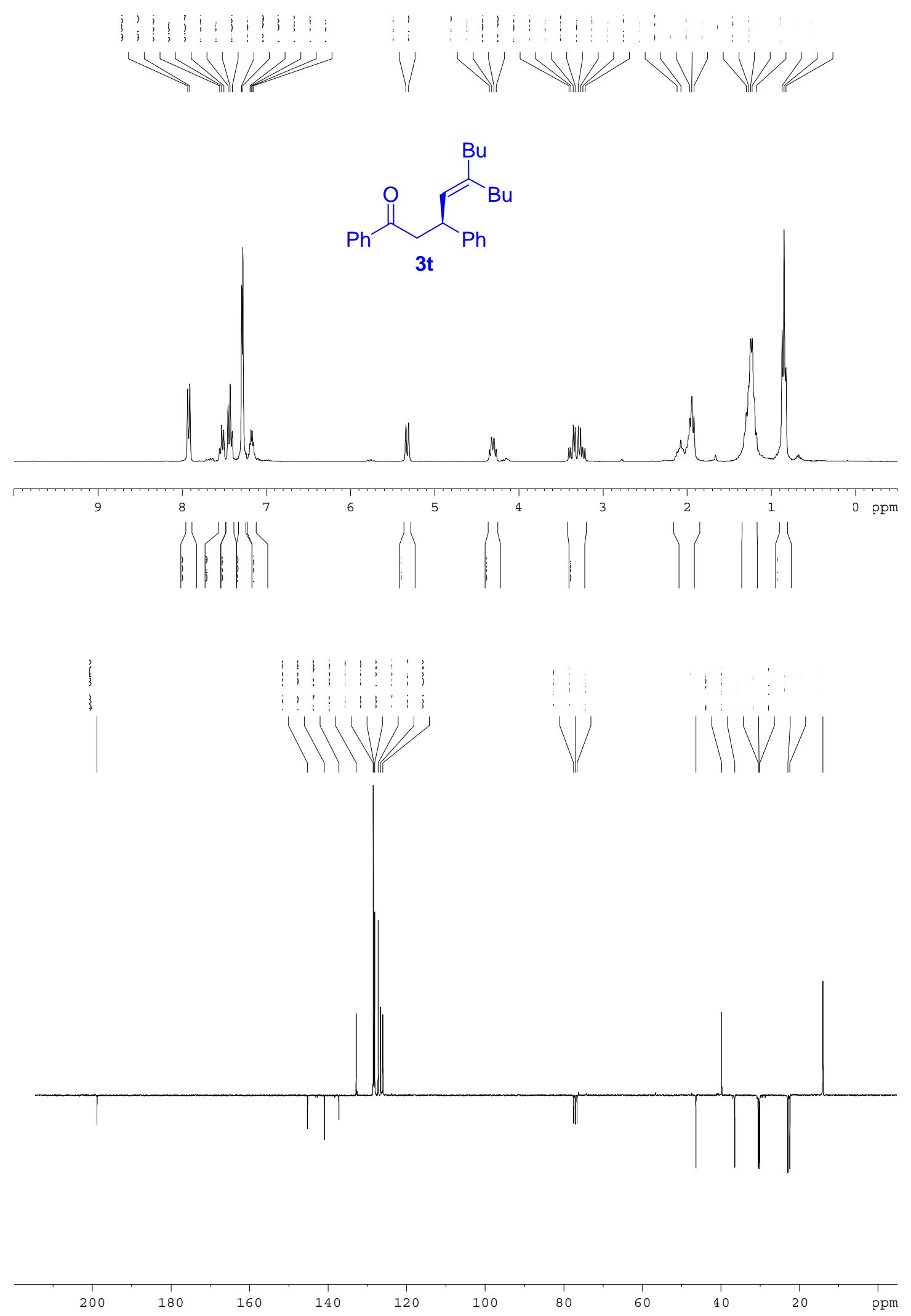



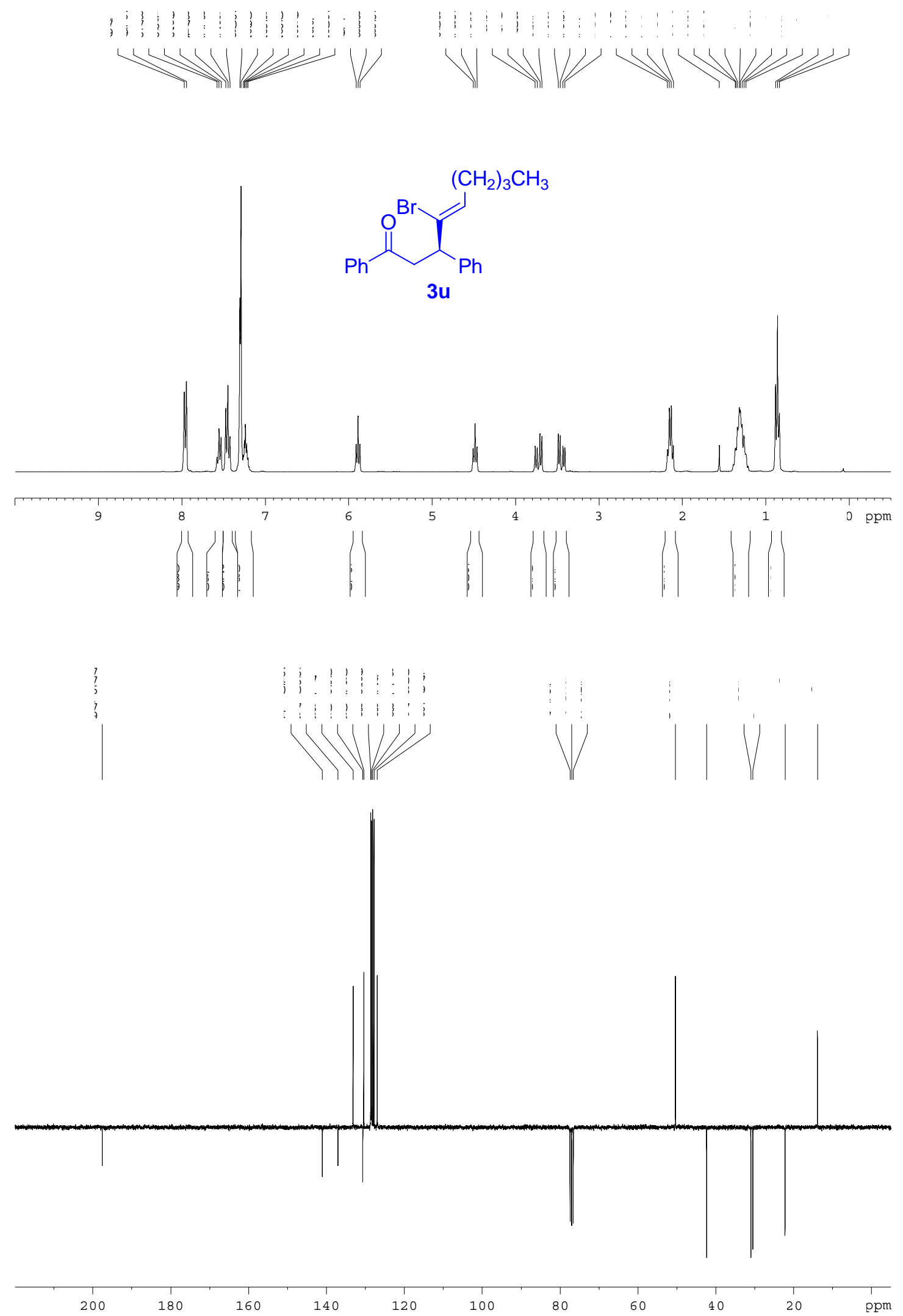